\title{
Daten zur Sozialstruktur der deutschen Abgeordneten des Europäischen Parlaments*
}

\author{
Peter Rütters
}

Obwohl das Europäische Parlament (EP) und die Gemeinsame Versammlung der EGKS, aus der das heutige Parlament der Europäischen Union hervorgegangen ist, inzwischen seit mehr als 60 Jahren bestehen, haben Analysen zur Sozialstruktur der Abgeordneten - und insbesondere der deutschen Mitglieder des EP - nur ein eher beiläufiges wissenschaftliches Interesse gefunden. Abgesehen von einer kurzlebigen Konjunktur nach Einführung der Direktwahl des EP im Jahr $1979^{1}$ hat sich die Aufmerksamkeit auf die Struktur, Kompetenzausstattung und Funktionen des Parlaments und auf dessen Rolle und Einflussmöglichkeiten in den Beziehungen zu den anderen Institutionen von EGKS, EWG, EG und schließlich EU konzentriert. ${ }^{2}$ Seit Einführung der Direktwahlen wurden Wahlberichte und Wahlanalysen ein beliebtes Forschungsfeld 3 , während die Abgeordnetensoziologie (Sozialstruktur-Analyse) nur beiläufig betrieben wurde. Es sollte daher nicht überraschen, dass den deutschen EuropaAbgeordneten ${ }^{4}$ - der größten nationalen Gruppe im EP - ein ähnlich sporadisches Interesse

* Die Untersuchungen zur "Sozialstruktur“ der deutschen Abgeordneten des Europäischen Parlaments und zum „Verbleib“ der aus dem EP ausgeschiedenen Parlamentarier (nachfolgender Beitrag in diesem Heft der ZParl) sind Vorstudien - und in gewisser Weise auch Machbarkeitsstudien - für ein geplantes komplexeres Forschungsprojekt, das neben den deutschen Abgeordneten des EP auch vergleichend Abgeordnete anderer EU-Staaten einbeziehen soll. Auch für diese liegen bisher keine systematischen Analysen der Sozialstruktur vor.

1 Vgl. Emil J. Kirchner, The European Parliament: Performance and Prospects, Aldershot 1984; Eva M. Thöne, Das direkt gewählte Europäische Parlament. Ein Beitrag zur Abgeordnetensoziologie, in: ZParl, 13. Jg. (1982), H. 2, S. 149 - 180; Richard Corbett / Francis Jacobs / Michael Shackleton, The European Parliament, London 2011, S. 51 - 64, sowie die vorangehenden sieben Ausgaben seit 1990; ferner Eberhard Grabitz / Otto Schmuck / Sabine Steppat / Wolfgang Wessels, Direktwahl und Demokratisierung - Eine Funktionsbilanz des Europäischen Parlaments nach der ersten Wahlperiode, Bonn 1988; die zuletzt angeführte Studie versucht zu zeigen, dass eine Bilanz des Europäischen Parlaments ohne differenzierte Sozialstruktur-Analyse der Abgeordneten auskommen kann, entsprechend auch die in der Auswahlbibliographie unter „B. 3. Der Abgeordnete“ (S. 690 f.) aufgelistete Literatur.

2 Vgl. für viele die Sammelbände: Andreas Maurer / Dietmar Nickel (Hrsg.), Das Europäische Parlament. Supranationalität, Repräsentation und Legitimation, Baden-Baden 2005; Jürgen Mittag (Hrsg.), 30 Jahre Direktwahlen zum Europäischen Parlament (1979 - 2009). Europawahlen und EP in der Analyse, Baden-Baden 2011; beide Sammelbände verzichten trotz einer thematischen Nähe auf eine Abgeordnetensoziologie.

3 Vgl. unter anderem Oskar Niedermayer / Hermann Schmitt (Hrsg.), Wahlen und Europäische Einigung, Opladen 1994; Forschungsgruppe Wahlen e.V., Europawahl. Eine Analyse der 4. Direktwahl zum Europaparlament 9. bis 12. Juni 1994, Mannheim 1994; Oskar Niedermayer, Die Wahl zum Europäischen Parlament vom 13. Juni 2004 in Deutschland: Ein schwarzer Tag für die SPD, in: ZParl, 36. Jg. (2005), H. 1, S. 3 - 19; ders., Die Wahl zum Europäischen Parlament vom 7. Juni 2009 in Deutschland: SPD-Debakel im Vorfeld der Bundestagswahl, in: ZParl, 40. Jg. (2009), H. 4, S. $711-731$.

4 Bis 1979 wurden die aus der Bundesrepublik Deutschland entsandten Abgeordneten vom Bundestag aus den Reihen der Bundestagsmitglieder delegiert; seit Einführung der Direktwahl wurden 78 Abgeordnete direkt gewählt und drei weitere Parlamentarier vom Berliner Abgeordnetenhaus bestimmt; seit 1994 wurden 99 Abgeordnete direkt gewählt. 
entgegengebracht wurde ${ }^{5}$, das durchaus der geringen Wahrnehmung dieser Parlamentarier in der Öffentlichkeit und bei den Wählern entspricht. ${ }^{6}$ Diese Diskrepanz zwischen Bevölkerung und Wahlbürgern auf der einen Seite und deren Repräsentanten, den Abgeordneten, und dem EP als einzigem direkt von der Bevölkerung legitimierten Organ der EU auf der anderen Seite hat verschiedene Gründe, die hier nur skizziert werden können:

- Sie resultiert nicht zuletzt aus der erst 1979 erfolgten Ablösung der von den nationalen Parlamenten delegierten Mitglieder durch direkt gewählte Abgeordnete; sie wurde aber noch dadurch gehemmt, dass erst 2002 vom Rat der EU ein Verbot der Doppelmitgliedschaft in nationalen Parlamenten und EP beschlossen wurde als weiterer Schritt zur personellen und institutionellen Eigenständigkeit des Parlaments der EU.7

- Dieses Defizit an Parlamentarisierung zeigt sich auch in dem zähen Ringen um ein europäisches Abgeordnetenstatut und der damit verbundenen einheitlichen und aus den Mitteln des EP zu erstattenden Abgeordnetenentschädigung. Da auch hier das EP nicht entscheidungsfähig ist, erfolgte eine Regelung erst mit dem Beschluss des Rates der EU im Jahr 2005, wirksam mit Beginn der 7. Wahlperiode (2009) - also 30 Jahre nach Einführung der Direktwahlen zum EP. ${ }^{8}$

- Mit der ungenügenden Parlamentarisierung korrespondiert - vor allem in den mitgliederstarken EU-Staaten - ein Defizit an Repräsentanz der Bürger im EP. Aufgrund einer degressiv gehaltenen Relation zwischen Bevölkerung und Abgeordneten` vertreten seit 1994 nur 99 Parlamentarier (1979 bis 1994: 81, davor 36) die Wähler und Einwohner der Bundesrepublik Deutschland. Bezogen auf die Bürgernähe und Responsivität von Politik ist diese Relation dürftig und führt dazu, dass Europa-Parlamentarier kaum bekannt sind oder bei populäreren Politikern ihr EP-Mandat nicht wahrgenommen wurde oder wird. ${ }^{10}$

- Diese Distanz zu den Mitgliedern des Europäischen Parlaments (MdEP) trägt mit dazu bei, dass die Wahlbeteiligung - nicht nur in Deutschland - in den dreißig Jahren, in denen Direktwahlen durchgeführt wurden, nahezu kontinuierlich gesunken ist und all-

5 Vgl. Karlheinz Reif/ Hermann Schmitt / Klaus Schubert, Wer sind und was wollen die Deutschen im Europäischen Parlament? Sozialprofil, politischer Rückhalt und Zielvorstellungen der deutschen Kandidaten zum Europäischen Parlament, in: ZParl, 10. Jg. (1979), H. 3, S. 332 - 354; Rudolf Hrbek / Carl-Christoph Schweitzer, Die deutschen Europa-Parlamentarier. Ergebnisse einer Befragung der deutschen Mitglieder des Europäischen Parlaments, in: APuZ, 39. Jg. (1989), B 3 , S. 3 - 18; für die Zeit vor der Direktwahl des EP siehe Peter Reichel, Bundestagsabgeordnete in europäischen Parlamenten. Zur Soziologie des europäischen Parlamentariers, Hamburg 1974.

6 Vgl. Oskar Niedermayer, Das Europäische Parlament in der öffentlichen Meinung - bekannt, aber wenig relevant, in: integration, 32. Jg. (2009), H. 3, S. 231 - 245; ferner ders., Die Wahl zum Europäischen Parlament, a.a.O. (Fn. 3), S. 721, Fn. 28.

7 Vgl. Peter Schindler, Datenhandbuch zur Geschichte des Deutschen Bundestages 1949 bis 1999, Band 1, Baden-Baden 1999, S. 468 f.; Michael F. Feldkamp, Datenhandbuch zur Geschichte des Deutschen Bundestages 1990 bis 2010, Baden-Baden 2011, S. 136; Jürgen Mittag, Wegmarke für die Parlamentarisierung der Europäischen Union: Die finanziellen Neuregelungen des europäischen Abgeordnetenhauses, in: ZParl, 37. Jg. (2006), H. 4, S. 713 - 728, S. 714, Fn. 14.

8 Vgl. Jürgen Mittag, a.a.O. (Fn. 7), S. 714.

9 Auf circa 820.000 Einwohner und 630.000 Wahlberechtigte kommt ein Abgeordneter; in den kleineren Ländern wie zum Beispiel Luxemburg ist diese Relation erheblich günstiger (etwa um den Faktor 10).

10 Zum Beispiel: Cem Özdemir (2004 bis 2009), Sarah Wagenknecht (2004 bis 2009), Lothar Bisky (2009 bis 2013), Reinhard Bütikofer (seit 2009) und so weiter. 
mählich eine Schwelle zu erreichen droht, die die Legitimation des EP in Frage zu stellen scheint. ${ }^{11}$ Mit dieser Entwicklung findet die nach wie vor plausible These eine Bestätigung, dass es sich bei den Europa-Wahlen überwiegend um „Sekundärwahlen“ handele, in denen nicht über europapolitische Fragen entschieden werde, sondern ,innerstaatliche Themen und parteipolitische Auseinandersetzungen der nationalen Ebene"12 im Vordergrund stünden.

- Schließlich verweisen die erwähnten Defizite darauf, dass es sich beim EP noch immer um ein Abgeordnetenhaus handelt, dessen Kompetenzen und Funktionen eher einem Parlament des Konstitutionalismus des 19. Jahrhunderts ähneln als der Volksvertretung einer repräsentativen Demokratie und eines parlamentarischen Regierungssystems der Gegenwart. Das EP mag als Parlament sui generis kategorisiert werden ${ }^{13}$, charakteristisch bleibt ein beachtlicher und in den Jahrzehnten der Direktwahl nur zögerlich abgebauter Kompetenzmangel im Vergleich zu nationalen Parlamenten wie dem Bundestag.

Von Bedeutung für die Wahrnehmung und Einordnung des EP als schwacher europapolitscher Akteur war dessen geringe Fähigkeit, den Prozess der Veränderung der supranationalen europäischen Strukturen von der EGKS bis zur Europäischen Union und damit verbunden auch die eigene Kompetenzausstattung in relevantem und erkennbarem Maße mitzugestalten. Diese Entwicklung wurde - ohne das EP - exekutiv vorbereitet (Kommission, nationale Regierungen) und nationalstaatlich (Parlamente, Referenden) legitimiert. Zwar kennzeichnen die Entwicklung des EP im Verlauf der Vertragsrevisionen seit dem Maastricht-Vertrag von 1992 ein bemerkenswerter Kompetenzzuwachs und eine Tendenz zur Parlamentarisierung der Entscheidungsprozesse in der EU. Als Defizit gilt hingegen die unzureichende „demokratische Repräsentation durch das EU-Parlament“, da es an einer notwendigen „europäischen Öffentlichkeit“ mangelte ${ }^{14}$ und die Responsivität des politischen Handelns der Abgeordneten die (Wahl-)Bürger nicht erreichte.

Auch die Abgeordneten des EP teilen mit den Mitgliedern anderer demokratischer Parlamente die konkurrierenden Anforderungen an ein Mandat. Es handelt sich um ein Mandat auf Zeit: das Ausscheiden aus dem Parlament und die Wahl neuer Abgeordneter gehören zu den zentralen Funktionsbedingungen eines repräsentativen demokratischen Systems. Die durch Wahlen erlangte Legitimation ist zeitlich limitiert und periodisch einer Erfolgskontrolle unterworfen. Diese Zeitbegrenzung konkurriert jedoch mit den Anforderungen eines funktions- und aufgabendifferenzierten Parlaments, die eine Professionalisierung der Abgeordneten verlangen, so dass zumindest ein Teil von ihnen in der Lage ist, länger als nur eine Wahlperiode dem Parlament anzugehören. Schließlich liegt es im materiellen und politischen Eigeninteresse der meisten Parlamentarier, die Politik zum Beruf gemacht haben, die Mandatsdauer so weit wie möglich zu verlängern.

11 Vgl. Andreas Biefang, Wie demokratisch ist die Europäische Union? Sechs Thesen aus parlamentarismusgeschichtlicher Perspektive, in: Jürgen Mittag (Hrsg.), a.a.O. (Fn. 2), S. 51 - 61, S. 51.

12 Jürgen Mittag / Claudia Hülsgen, Von Sekundärwahlen zu europäisierten Wahlen? 30 Jahre Direktwahlen zum Europäischen Parlament, in: integration, 32. Jg. (2009), H. 2, S. 105 - 122, S. 107; ferner Andreas Maurer / Wolfgang Wessels, Das Europäische Parlament nach Amsterdam und Nizza: Akteur, Arena oder Alibi?, Baden-Baden 2003, S. 179 - 183.

13 Vgl. Stefan Marschall, Zwischen Völker- und Bürgervertretung: Das EP und die Europawahlen im Spannungsfeld repräsentativer Demokratie jenseits des Nationalstaates, in: Jürgen Mittag (Hrsg.), a.a.O. (Fn. 2), S. 33 - 49.

14 Andreas Biefang, a.a.O. (Fn. 11), S. 58. 
Im Folgenden sollen Daten zur Sozialstruktur, zur Verweildauer sowie zur Parlaments- und Regierungserfahrung der deutschen EP-Abgeordneten in Form komprimierender Tabellen und knapper Kommentierung vorgestellt werden, und zwar für die sieben Wahlperioden seit der ersten Direktwahl im Jahr 1979. Die Dokumentation und die Tabellen stützten sich vor allem auf die biographischen Daten in den Handbüchern des EP. 15

\section{Altersstruktur}

Es wäre nicht überraschend, wenn sich die Altersstruktur der Mitglieder des ersten direkt gewählten EP erheblich von der der folgenden Wahlperioden unterschiede. Nicht nur gehörten 16 Abgeordnete (19,8 Prozent des neugewählten EP) bereits dem vorherigen EP an und waren folglich auch Mitglieder des Bundestages. Darüber hinaus setzte sich bei der ersten Direktwahl die bisherige Praxis fort, Bundestagsabgeordnete weiterhin (letztlich alternativ) für ein Mandat im EP zu nominieren. Zu den erwähnten $16 \mathrm{MdEP}$, die bereits vor der Direktwahl einen Sitz in beiden Parlamenten hatten, kamen noch 19 neue EP-Abgeordnete, die noch Mitglied des Bundestages waren oder dies früher gewesen waren. ${ }^{16}$ Bei der ersten Direktwahl waren die Parteien bemüht, politisch und gesellschaftlich bekannte und angesehene Personen für eine Kandidatur zu gewinnen, um eine hohe Wahlbeteiligung und eine öffentliche Akzeptanz des EP zu erreichen. ${ }^{17}$ Eine Folge dieser Nominierungs- und Wahlbesonderheiten zeigt sich in einem - im Vergleich zu den meisten folgenden Wahlperioden - etwas höheren Anteil von Abgeordneten, die 50 Jahre und älter waren (53,2 Prozent). Überraschend - im Vergleich mit der Altersstruktur in den folgenden Wahlperioden - ist jedoch der hohe Anteil der Altersgruppe 30 bis 39 Jahre (21 Prozent), während die

15 Vgl. Amtliches Handbuch des Europäischen Parlaments 1979 - 1984, 1. Wahlperiode, herausgegeben vom Generalsekretariat des Europäischen Parlaments, Luxemburg 1980 (Loseblatt-Sammlung); Amtliches Handbuch des Europäischen Parlaments 1984, 2. Wahlperiode 1984 - 1989, herausgegeben vom Generalsekretariat des Europäischen Parlaments, Luxemburg 1985 (Stand: 1. Dezember 1984); Die Mitglieder des Europäischen Parlaments, 3. Wahlperiode 1989 - 1994, herausgegeben vom Generalsekretariat des Europäischen Parlaments, Luxemburg 1990 (Stand: April 1990); Die Mitglieder des Europäischen Parlaments, 4. Wahlperiode 1994 - 1999, herausgegeben vom Generalsekretariat des Europäischen Parlaments, Luxemburg 1996 (Stand: Februar 1996); Die Mitglieder des Europäischen Parlaments, 5. Wahlperiode 1999 - 2004, herausgegeben vom Generalsekretariat des Europäischen Parlaments, Luxemburg 2001 (Stand: Juni 2001); Europäisches Parlament: Bürger-Handbuch, herausgegeben von Klaus Löffler, Leiter des deutschen Informationsbüros des Europäischen Parlaments in Deutschland, 6. Wahlperiode 2004 - 2009, Berlin 2005 (Stand: Februar 2005); Europäisches Parlament: Bürger-Handbuch, herausgegeben von Frank Piplat, Leiter des deutschen Informationsbüros des Europäischen Parlaments in Deutschland, 6. Wahlperiode 2009 - 2014, Berlin 2012 (Stand: April 2012).

16 Nur sieben von diesen 19 Abgeordneten hatten 1979/80 kein Bundestagsmandat. Zur Veränderung des Anteils der MdEP, die vor ihrem Europa-Mandat Mitglied des Bundestages waren, vgl. Tabelle 8.

17 Die Nominierung von Willy Brandt, Philipp von Bismarck, Katharina Focke, Alfons Goppel, Otto von Habsburg-Lothringen, Wilhelm F.T. Hahn, Kai Uwe von Hassel, Karl Hauenschild, Heinz Kühn, Eugen Loderer, Heinz Oskar Vetter, Hans Katzer, Casimir J. Prinz zu Sayn-Wittgenstein-Berleburg dürfte überwiegend mit dieser Intention erfolgt sein und erfüllte hinsichtlich der Wahlbeteiligung diesen Zweck. 
Altersgruppe der 40- bis 49-Jährigen schwächer in der 1. Wahlperiode vertreten war (vgl. Tabelle 1). 18

In der Altersgruppe unter 30 Jahren finden sich in allen Wahlperioden nur wenige Abgeordnete, da eine Nominierung für ein EP-Mandat in der Regel eine längere Parteimitgliedschaft, Ausübung innerparteilicher Funktionen, meistens auch kommunalpolitische Mandate und Ämter, nicht selten ein Bundestags- oder Landtagsmandat sowie eine gewisse europapolitische Affinität voraussetzt. ${ }^{19}$ Nicht nur die vielfältigen und intensiven Anforderungen des Parlamentsalltags dürften die geringe Anzahl von MdEP in der Altersgruppe 70 Jahre und älter und auch die in fast allen folgenden Wahlperioden geringe Anzahl von Abgeordneten, die in der Altersgruppe 60 bis 69 Jahre neu in das EP gewählt wurden, erklären. Nach der 1. Wahlperiode nahm die Bereitschaft der Parteien ab, mit der Nominierung erfahrener und bekannter Landes- und Bundespolitiker für die Europa-Wahl und für die eigene Partei zu werben sowie verdiente Politiker mit langer parlamentarischer und Regierungserfahrung zum Abschluss ihrer politischen Karriere mit einem Mandat im EP zu honorieren. ${ }^{20}$

Tabelle 1 zeigt an, dass der Altersschwerpunkt der Abgeordneten in der Gruppe der 50bis 59-Jährigen liegt, sich aber während der sieben Wahlperioden auch in den beiden angrenzenden Altersgruppen der 40- bis 49-Jährigen und der 60- bis 69-Jährigen ein hoher, manchmal auch höherer Anteil von Abgeordneten findet. Fasst man jeweils zwei Altersgruppen zusammen (30 bis 49, 40 bis 59, 50 bis 69), wird deutlich, dass die Mehrzahl der Abgeordneten - was nicht überraschen wird - in allen Wahlperioden, wenngleich mit beachtlichen Schwankungen (56,8 Prozent in der 1. Wahlperiode, 76,8 Prozent in der 4. und 5. Wahlperiode) in der mittleren Gruppe (40 bis 59 Jahre) zu finden ist. In diesem Befund kommen zwei sich einander verstärkende Faktoren zum Ausdruck: die oben erwähnten Anforderungen und Voraussetzungen für parteiinterne Kandidatenaufstellungen sowie die sich häufig über mehrere Wahlperioden erstreckende Mitgliedschaft im EP.

Über die kleineren Parteien (FDP, Grüne, PDS / Die Linke) ${ }^{21}$ können angesichts der geringen Anzahl von Abgeordneten (überwiegend jeweils weniger als zehn und höchsten 14

18 Das über alle Wahlperioden etwa gleiche Durchschnittsalter der Abgeordneten findet für die 1. Wahlperiode hierin eine Erklärung.

19 Vgl. Rudolf Hrbek / Carl-Christoph Schweitzer, a.a.O. (Fn. 5), S. 4 f. Zu den Fragen, wie Parteien ihre Abgeordneten rekrutieren, wer die Bewerber um eine Nominierung sind und wer sie unter welchen Kriterien auswählt vgl. Benjamin Höhne, Rekrutierung von Abgeordneten des Europäischen Parlaments. Organisation, Akteure und Entscheidungen in Parteien, Opladen 2013.

20 Von den zwölf Abgeordneten, die in der 1. Wahlperiode neu in das EP gewählt wurden und 60 Jahre und älter waren, können mindestens acht als politisch und öffentlich bekannt eingeordnet werden; in der 3. Wahlperiode waren es nur noch zwei von sechs Abgeordneten, in der 4. Wahlperiode war es nur ein Abgeordneter (Wolfgang Ullmann, Die Grünen), der mit über 60 Jahren neu ins EP gewählt wurde und als prominent gelten kann; in der 5 . Wahlperiode war es einer von zwei Abgeordneten (Hans Modrow, PDS); in der 6. Wahlperiode war es einer von vier Abgeordneten (der Unternehmer Vural Öger, SPD); schließlich in der 7. Wahlperiode zwei von vier Abgeordneten (Lothar Bisky, Die Linke; Birgit Schnieber-Jastram, CDU). Die Mehrzahl der Abgeordneten, die zu Beginn der jeweiligen Wahlperiode 60 Jahre und älter waren, gehörten seit der 2. Wahlperiode dem EP zumindest bereits in der vorhergehenden Wahlperiode an und waren mit ihrem Mandat in diese Altersgruppe vorgerückt.

21 Die nur in der 3. Wahlperiode (1989 bis 1994) mit sechs Abgeordneten im EP vertretene Partei REP kann hier unberücksichtigt bleiben. 


\begin{tabular}{|c|c|c|c|c|c|c|c|c|c|}
\hline & 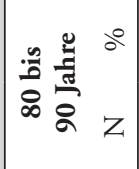 & 1 & 1 & 1 & & $\begin{array}{l}1 \\
1\end{array}$ & 1 & 1 & 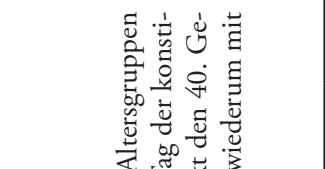 \\
\hline & 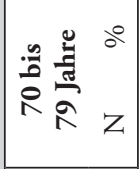 & $\begin{array}{l}\tilde{i} \\
\sim\end{array}$ & & & $\begin{array}{l}1 \\
1\end{array}$ & $\stackrel{\circ}{\rightarrow}$ & 1 & 1 & 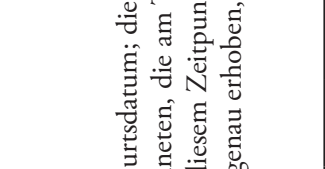 \\
\hline & 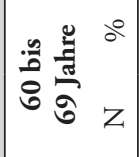 & $\stackrel{\infty}{\varrho}$ & $\begin{array}{l}0 \\
\infty\end{array}$ & $\begin{array}{l}\stackrel{2}{=} \\
\stackrel{2}{\beth}\end{array}$ & $\begin{array}{l}\overrightarrow{6} \\
0\end{array}$ & $\begin{array}{l}\overrightarrow{0} \\
0\end{array}$ & & $\stackrel{\sim}{\sim}$ & 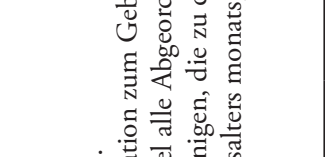 \\
\hline 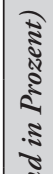 & 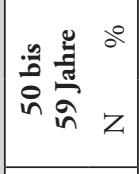 & $\hat{\tilde{n}}$ & $\stackrel{m}{m}$ & $\stackrel{\sim}{\hat{\imath}}$ & $\begin{array}{l}\stackrel{F}{F} \\
F\end{array}$ & $\begin{array}{l}\stackrel{+}{\circ} \\
\text { \&े } \\
\text { 암 }\end{array}$ & $\begin{array}{l}\stackrel{H}{\circ} \\
\stackrel{+}{+}\end{array}$ & $\hat{n}$ & 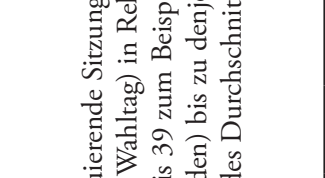 \\
\hline 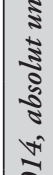 & 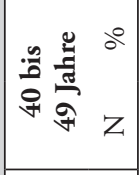 & $\hat{\sim}$ & $\hat{\hat{o}}$ & $\stackrel{0}{\stackrel{f}{\sharp}}$ & $\begin{array}{l}\sqrt[w]{n} \\
n \\
n\end{array}$ & $\begin{array}{l}\stackrel{\forall}{\delta} \\
i n \\
i n\end{array}$ & $\begin{array}{l}\tilde{\imath}^{2} \\
\approx\end{array}$ & 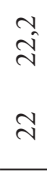 & 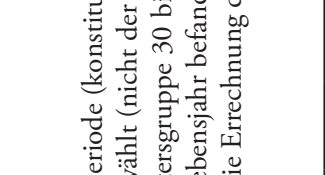 \\
\hline 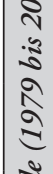 & 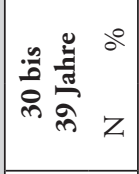 & $\stackrel{\circ}{\vec{v}}$ & $\begin{array}{l}\overrightarrow{=} \\
a\end{array}$ & $\begin{array}{l}\text { ? } \\
\stackrel{1}{0}\end{array}$ & $\begin{array}{l}\overrightarrow{ \pm} \\
\pm\end{array}$ & $\begin{array}{l}\infty \\
\infty\end{array}$ & & $\overrightarrow{ \pm}$ & 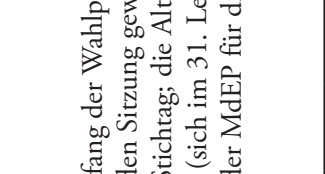 \\
\hline$\frac{\sqrt{2}}{\sqrt[3]{2}}$ & 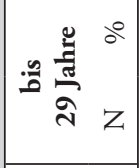 & 1 & $\hat{\imath}$ & 1 & $\begin{array}{l}\text { i } \\
\text { in }\end{array}$ & $\begin{array}{l}\stackrel{\circ}{+} \\
\stackrel{+}{*}\end{array}$ & $\stackrel{\circ}{\forall}$ & $n$ & 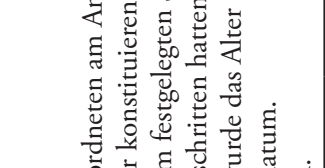 \\
\hline 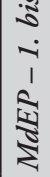 & 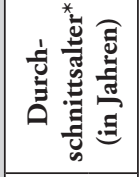 & $\hat{\tilde{n}}$ & $\stackrel{2}{\stackrel{\sigma}{\sigma}}$ & $\hat{n}$ & $\vec{\sigma}$ & $\stackrel{\circ}{\mathscr{q}}$ & $\stackrel{\forall}{\circ}$ & $\begin{array}{l}0 \\
\vec{n}\end{array}$ & 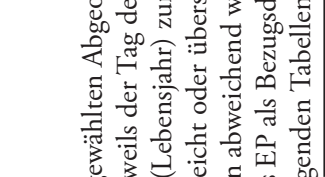 \\
\hline है & Z & 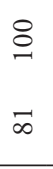 & 8 & $\stackrel{8}{8}$ & 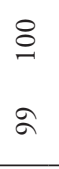 & 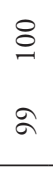 & 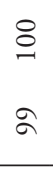 & aे & 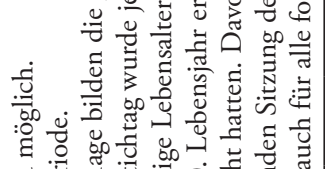 \\
\hline 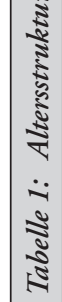 & & 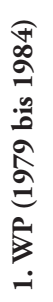 & 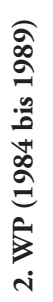 & 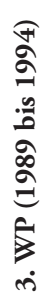 & 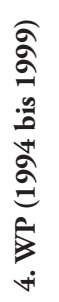 & 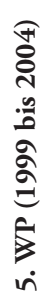 & 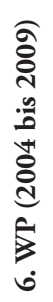 & 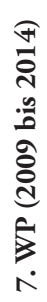 & 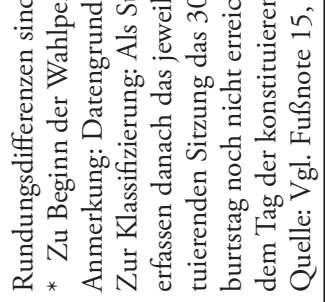 \\
\hline
\end{tabular}


Mandate) keine verallgemeinerbaren Aussagen über die Altersverteilung und ihre Veränderung im Verlauf von sieben Wahlperioden gemacht werden. ${ }^{22}$ Nur für die CDU/CSU- und die SPD-Abgeordneten lassen sich einige Daten zur Altersstruktur und deren Veränderung präsentieren, die nicht bereits durch Variationen von zwei oder drei Abgeordneten markant beeinflusst würden (vgl. Tabelle 2). In etwa weisen beide Parteien einen überwiegend ähnlichen Altersschwerpunkt auf. Die Mehrzahl ihrer Abgeordneten gehört(e) den Altersgruppen zwischen 40 und 59 Jahren an. Darüber hinaus finden sich noch stetig Abgeordnete sowohl in der jüngeren Altersgruppe 30 bis 39 Jahre (was als Potential für Erneuerung und „Verjüngung" verstanden werden kann) als auch in der Altersgruppe 60 bis 69 Jahre, die insgesamt etwas stärker vertreten ist.

In diesem Befund zeigt sich wiederum das Zusammenwirken der zwei bereits benannten Faktoren: der altersmäßig „späte“ Einstieg in das EP und die häufig zwei und mehr Wahlperioden umfassende Verweildauer. Eine Konzentration in den Altersgruppen 40 bis 59 ist bei der SPD ausgeprägter als bei der CDU/CSU. Dies dürfte für die Sozialdemokraten aus der geringeren Anzahl der Parlamentsmandate in allen Wahlperioden und insbesondere aus dem fast kontinuierlichen Rückgang der Parlamentssitze seit der 4. Wahlperiode resultieren, was die Möglichkeiten für eine „Verjüngung“ der Abgeordneten begrenzte.

Auch das EP erwies sich nicht als Vorreiter für die Präsenz von Frauen. ${ }^{23}$ Der Anteil weiblicher Abgeordneter erreichte erst in der 3. Wahlperiode (1989 bis 1994) mit 30,7 Prozent (25 MdEP) ein Niveau (vgl. Tabelle 7), das einige - zurückhaltende - Aussagen über die Altersstruktur erlaubt. Seitdem zeigte sich auch bei den Parlamentarierinnen, mit nur geringen Unterschieden zu allen Abgeordneten, ein Schwerpunkt in den Altersgruppen 40 bis 59 Jahre, in denen zwischen 64,8 Prozent (7. Wahlperiode) und 81,1 Prozent (5. Wahlperiode) der Mandatsträgerinnen am Anfang der jeweiligen Wahlperiode vertreten sind (vgl. Tabelle 3).

Ohne hier auf die vielfältigen Interpretationsmöglichkeiten im Detail eingehen zu wollen, verweist die Tabelle 4, die die Abgeordneten nach Geburtsjahrgängen „sortiert“, auf Veränderungen der politischen Sozialisierung der Parlamentarier im zeitlichen Verlauf. Hervorzuheben ist zunächst, dass Ende der 70er / Anfang der 80 Jahre (in der 1. Wahlperiode) jene Abgeordneten eine Mehrheit bildeten, deren politische Sozialisierung in der Zeit der Weimarer Republik und / oder des Nationalsozialismus erfolgt war. Der Rückbezug auf die Zeiten der politischen Sozialisationsprozesse der Abgeordneten und deren langsame, vielleicht sogar „verzögerte“ Ablösung aufgrund der langen EP-Mitgliedschaft lassen sich indes nicht auf einen „politischen Nenner“ bringen. Die gleichzeitig - aber nicht in gleicher Weise - erlebten historisch-politischen Ereignisse dürften nicht zu ähnlichen politischen und

22 Die FDP, die in vier Wahlperioden im EP vertreten war, hatte in der 1. Wahlperiode vier Abgeordnete, die auf zwei Altersgruppen verteilt waren; in der 3. Wahlperiode waren wiederum vier MdEP vertreten, diesmal in drei Altersgruppen; in der 6. Wahlperiode verfügten die Liberalen über sieben Abgeordnete, verteilt auf nunmehr vier Altersgruppen; ebenfalls auf vier Altersgruppen sind die nunmehr zwölf Abgeordneten der 7. Wahlperiode verteilt. Mit Ausnahme der 7. Wahlperiode gehörten jeweils ein bis drei Abgeordnete einer Altersgruppe an; eine Veränderung von einem Abgeordneten bedeuten Sprünge von 8,3, 14,3 beziehungsweise 25,0 Prozentpunkten, was keine verlässlichen Tendenz- oder Trendaussagen erlaubt. Für die anderen kleinen Parteien gilt mit geringen Variationen das Gleiche.

23 Vgl. den Abschnitt zur Geschlechterzusammensetzung. 


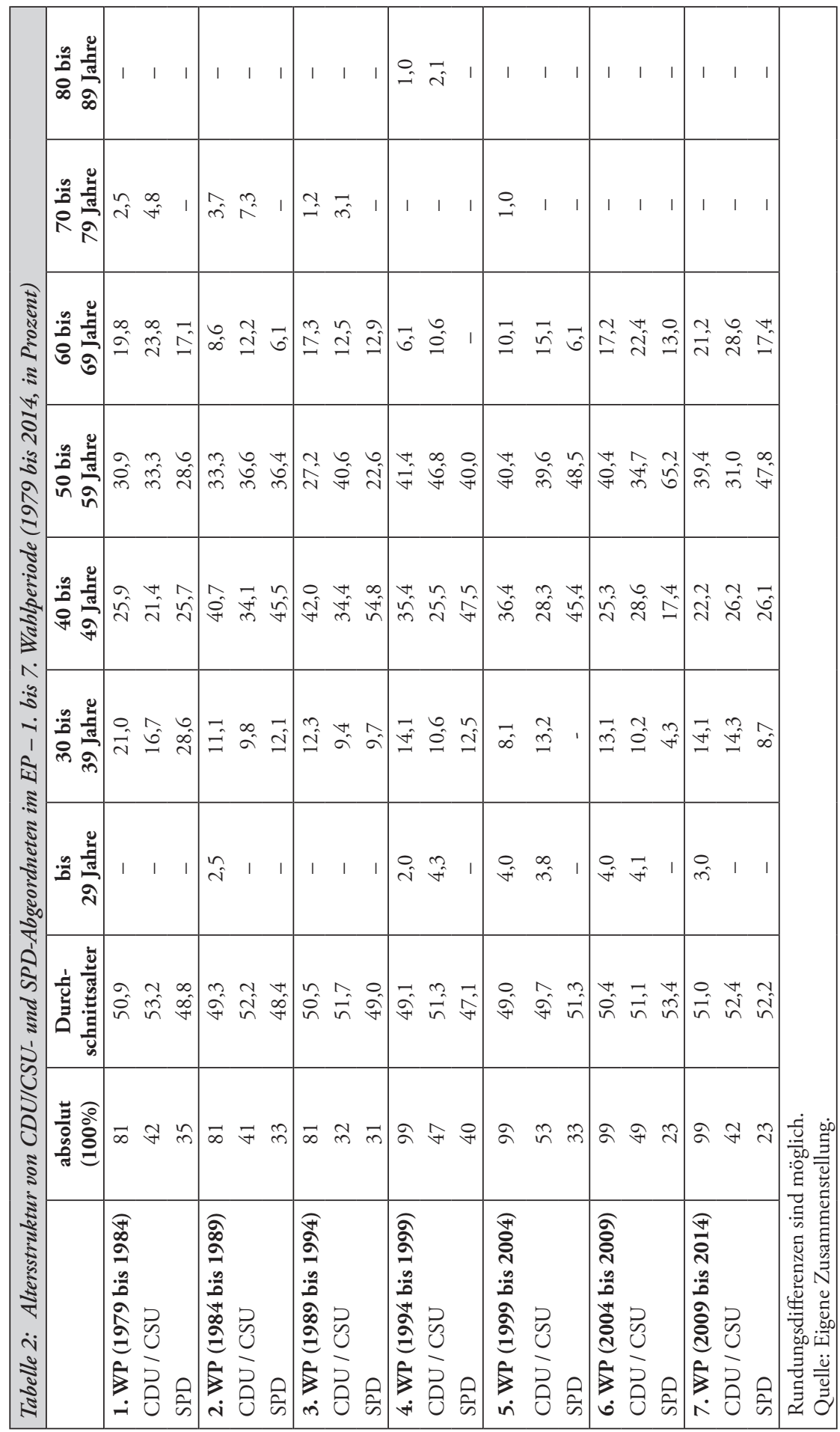




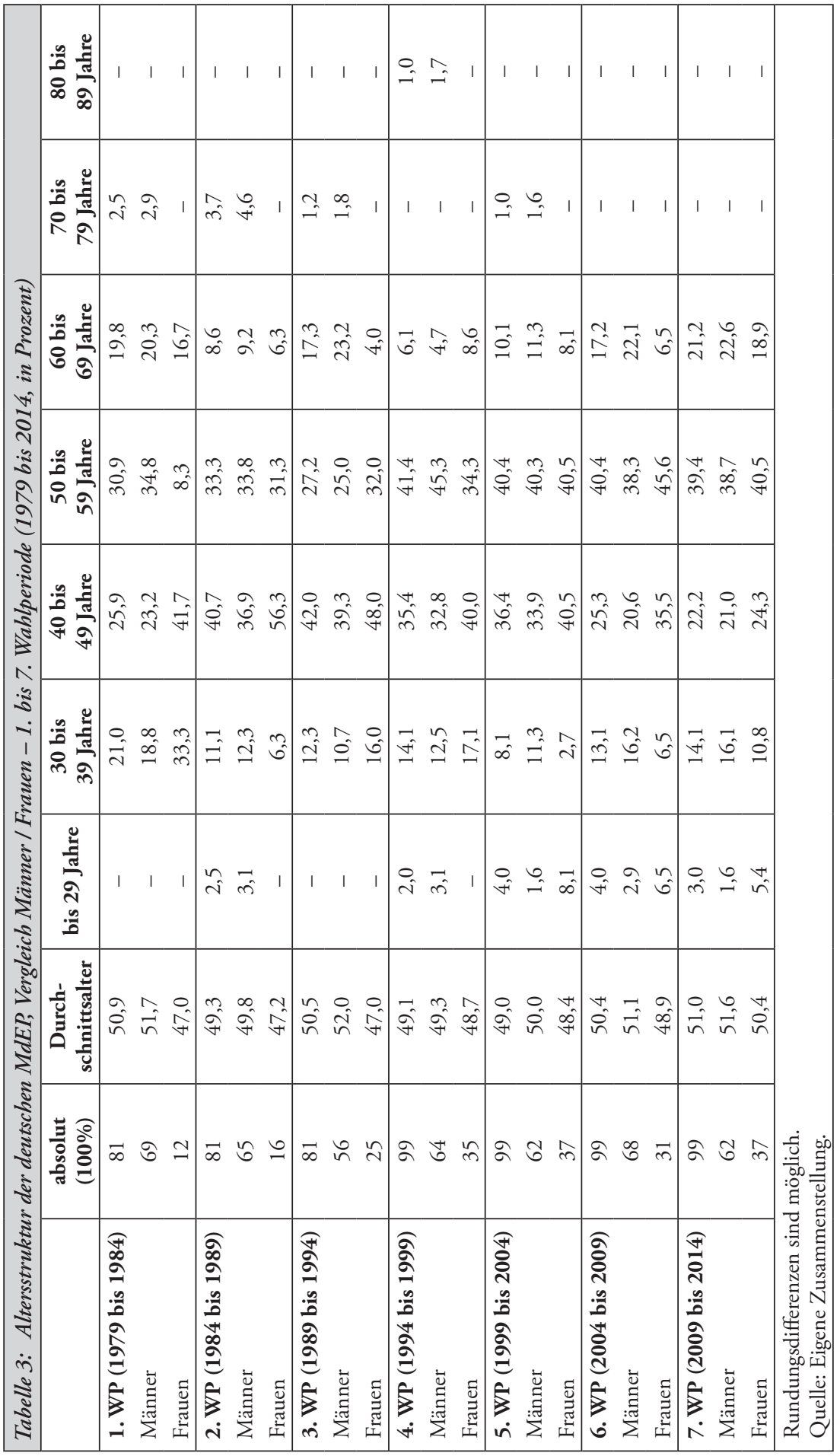


gesellschaftspolitischen Einstellungen geführt haben. ${ }^{24}$ Ein weiterer Aspekt, auf den diese Tabelle hinweist, ist die lange Zeit, in der einzelne Geburtsjahrgangsgruppen im EP präsent waren und sind.

\section{Bildungsabschlüsse}

Tabelle 5 zeigt ein sehr hohes formales Schulbildungsniveau der deutschen MdEP. Die Hochschulreife wurde kontinuierlich von circa 80 Prozent der Parlamentarier als höchster Bildungsabschluss erreicht (die Schwankungen um diesen Wert sind von geringer Aussagekraft). Ausschließlich einen Volks-/Grundschulabschluss hatten seit der 1. Wahlperiode nur wenige Abgeordnete, und dies findet sich in den letzten Wahlperioden auch nur noch vereinzelt. In diesem Befund spiegeln sich Erwartungen an sprachliche und interkulturelle Kompetenzen von Europa-Abgeordneten, vor allem aber eine allgemeine - und in Parlamenten verstärkt vertretene - Tendenz zu höherer Schul- und Ausbildung. Diese Entwicklung kommt auch in dem hohen Anteil von Abgeordneten zum Ausdruck, die ein Studium begonnen und überwiegend mit qualifizierendem Examen abgeschlossen haben. ${ }^{25}$

24 Das lässt sich exemplarisch an einigen biographischen Angaben von Abgeordneten des ersten direktgewählten Europäischen Parlaments verdeutlichen, ohne diese Selbstaussagen hier kommentieren zu wollen (Quelle: Amtliches Handbuch des Europäischen Parlaments 1979 - 1984, a.a.O. (Fn. 15): von Heinrich Aigner (geboren 1924) findet sich die Angabe: „1943 bis 1945 Kriegsteilnehmer“ (S. 6); von Philipp von Bismarck (geboren 1913): „Landwirtschaftliche Ausbildung; ab 1935 Wehrdienst, Kriegsteilnahme“ (S. 34a); von Erik Bernhard Blumenfeld (geboren 1915): „Bis 1939 kaufmännische und sprachliche Ausbildung, sowie Studium an der TH Berlin. Abbruch durch Kriegsteilnahme. 1942 Verhaftung und Überbringung in die KZ Auschwitz und Buchenwald." (S. 36); von Isidor W. Früh (geboren 1922): „1941 bis 1947 Wehrdienst und Gefangenschaft“ (S. 141); von Karl Fuchs (geboren 1920): „1940 bis 1943 Wehrdienst (Schwerkriegsbeschädigt)“ (S. 142); von Alfons Goppel (geboren 1905): „Studium der Rechts- und Staatswissenschaft in München, Rechtsanwalt, Staatsanwalt; 1939 bis 1945 Kriegsteilnehmer" (S. 161); von Kai Uwe von Hassel (geboren 1913): „1919 bis 1935 in Deutschland: Abitur, Ausbildung; 1935 bis 1939 Pflanzer in Ostafrika, bei Kriegsausbruch Ausweisung; 1940 bis 1945 Kriegsdienst" (S. 176); von Wilhelm Helms (geboren 1923): „1942 Abitur, bis 1945 Kriegsdienst“ (S. 177); von Heinz Kühn (geboren 1912): „Studium der Nationalökonomie und Staatswissenschaften; 1933 bis 1945 Emigration nach der Tschechoslowakei und Belgien“ (S. 214); von Erwin Lange (geboren 1914): „Seit 1928 aktiv in der sozialistischen Jugendbewegung, seit 1930 in der Gewerkschaftsbewegung tätig. 1936 aus politischen Gründen verhaftet, 1937 wegen Vorbereitung zum Hochverrat zu mehrjähriger Zuchthausstrafe und Ehrverlust verurteilt, Moorarbeit. Danach Schutzhaft und Polizeiaufsicht. Im Herbst 1942 zur Strafeinheit 999 eingezogen, Einsatz in Afrika. 1943 bis 1946 Kriegsgefangenschaft in den USA.“ (S. 217); von Horst Lange (geboren 1928): „Mit 15 Jahren Kriegsdienst, verwundet, Gefangenschaft.“ (S. 218); von Wolfgang Schall (geboren 1916): „Humanistisches Abitur; 1934 bis 1945 Berufssoldat, seit 1943 Major i.G., 1945 bis 1955 Kriegsgefangenschaft in der Sowjetunion (...) 1957 Eintritt in die Bundewehr. Tätigkeit in der NATO“ (S. 339). Eine Anzahl von Abgeordneten dieser Altersgruppe machte keine Angaben über diese Phase ihres Lebens.

25 Diese Einschränkung muss deshalb gemacht werden, weil bei einigen Abgeordneten die Angaben in den ausgewerteten Kurzbiographien der Parlamentshandbücher nicht eindeutig sind. Aus pragmatischen Gründen wurde daher in dieser Kategorie jedes erwähnte Studium erfasst, selbst wenn erkennbar war, dass es nicht abgeschlossen wurde. Für die weit überwiegende Mehrheit der Abgeordneten konnte indes festgestellt werden, dass sie das Studium qualifiziert beendete. 


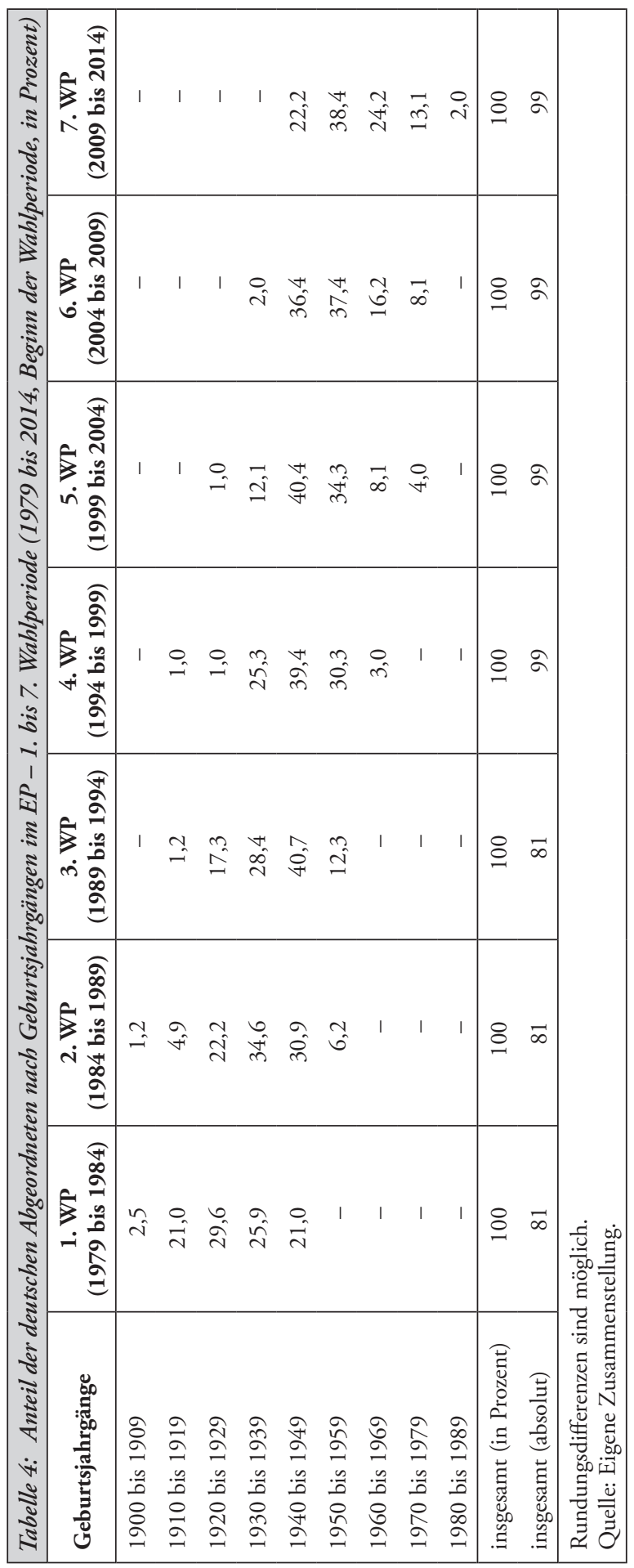




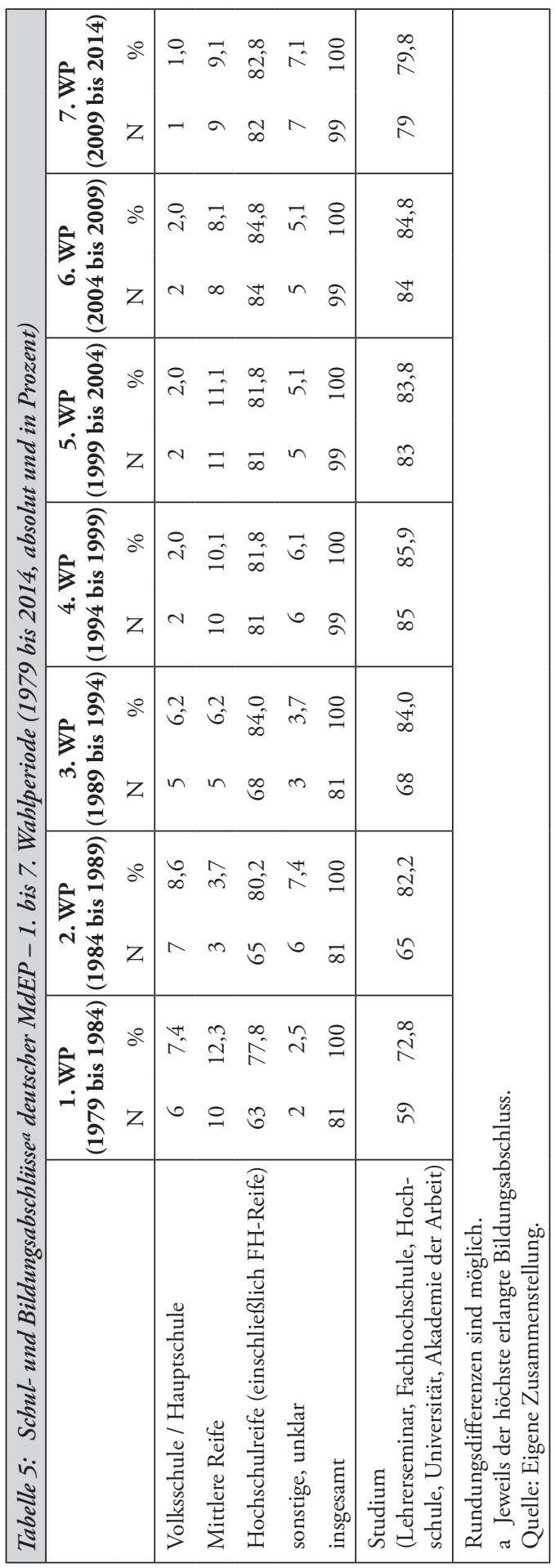


Eine Aufschlüsselung der Qualifikationsstruktur auf die Parteien, die in Tabelle 6 für die 1 . und die 7. Wahlperiode dargestellt wird, zeigt für die kleinen Parteien (FDP, Die Grünen, PDS / Die Linke) eine geringe Abweichung von den Durchschnittswerten. Im Vergleich dazu verfügt nur bei den sozialdemokratischen Abgeordneten ein deutlich größerer Teil der Parlamentarier nicht über die Hochschulreife. Folglich ist auch der Anteil der Abgeordneten, die kein Studium absolvierten, etwas größer. Für die 1. Wahlperiode resultierte der relativ hohe Anteil von Abgeordneten mit Volkschulbildung und Mittler Reife aus einer Anzahl von Gewerkschaftern und Betriebsräten, die aufgrund ihrer Funktionen in den Organisationen zu einflussreichen politischen Positionen bis hin zu einem Mandat im EP gelangten. In der 7. Wahlperiode sind die Gründe für den etwas geringeren Anteil von Abgeordneten mit Hochschulreife und einem Studium indes diffuser. Doch auch für die hier hervorgehobenen sozialdemokratischen Abgeordneten ist festzuhalten, dass sie insgesamt - wie die Parlamentarier der anderen Parteien - über ein hohes Schulbildungs- und Ausbildungsniveau (Studium) verfügen.

\section{Geschlechterzusammensetzung}

Frauen sind auch unter den deutschen Abgeordneten des EP bis in die Gegenwart unterrepräsentiert im Hinblick auf die Geschlechterproportion der Bevölkerung. Das sollte nicht verwundern, da die direktgewählten Parlamentarierinnen von den Parteien nominiert werden, die auch für Landtags- und Bundestagswahlen die Kandidatinnen aufstellen beziehungsweise nicht aufstellen und auf sichere oder eher auf unsichere Listenplätze positionieren. Tabelle 7 zeigt die Entwicklung der Geschlechterzusammensetzung für die sieben Wahlperioden des EP. Auf den ersten Blick ist zu erkennen, dass sich im Laufe der ersten drei Wahlperioden der Anteil von Frauen von 14,8 Prozent auf beinahe beeindruckende 30,7 Prozent mehr als verdoppelt hat, um dann allmählich - mit Ausnahme der 6. Wahlperiode - auf das Niveau von 37,3 Prozent zu klettern.

Immerhin lag bei der SPD der Frauenanteil seit der 1. Wahlperiode kontinuierlich über dem Durchschnitt, während die zusammengefassten Daten von CDU und CSU mit gleicher Regelmäßigkeit bis in die Gegenwart erheblich unterhalb dieses Mittelwertes rangieren. In beiden Parteien finden sich bei den Abgeordneten Geschlechterproportionen wieder, wie sie in ähnlicher Weise auch für den Bundestag festgestellt wurden. ${ }^{26}$ Bei den Grünen und der PDS / Die Linke wurde die grundsätzliche Entscheidung, mindestens die Hälfte der Mandate an Frauen zu vergeben, fast in allen Wahlperioden, in denen die Parteien im EP vertreten waren, umgesetzt (bei den Grünen mit Ausnahme der 2. Wahlperiode), während sich bei der FDP erst in der letzten Wahlperiode eine Bereitschaft zeigte, den Frauenanteil zu erhöhen.

26 Vgl. Peter Schindler, a.a.O. (Fn. 7), S. 636; Michael F. Feldkamp, a.a.O. (Fn. 7), S. 276; die Landesparlamente weisen hingegen noch immer beachtliche Variationen des Frauenanteils auf, vgl. Werner Reutter, Föderalismus, Parlamentarismus und Demokratie. Landesparlamente im Bundesstaat, Opladen 2008, S. $119 \mathrm{ff}$. 


\begin{tabular}{|c|c|c|c|c|c|}
\hline \multicolumn{6}{|c|}{$\begin{array}{l}\text { Tabelle 6: Schul- und Bildungsabschlïssea deutscher MdEP nach Parteien- } \\
\text { 1. und 7. Wahlperiode (1979 bis } 1984 \text { und } 2009 \text { bis 2014, absolut und in Prozent) }{ }^{b}\end{array}$} \\
\hline & & \multicolumn{2}{|c|}{ 1. WP (1979 bis 1984) } & \multicolumn{2}{|c|}{ 7. WP (2009 bis 2014) } \\
\hline & & $\mathrm{N}$ & $\%$ & $\mathrm{~N}$ & $\%$ \\
\hline Volksschule / Hauptschule & $\begin{array}{l}\text { CDU / CSU } \\
\text { SPD } \\
\text { FDP } \\
\text { Grüne } \\
\text { Linke } \\
\text { insgesamt }\end{array}$ & $\begin{array}{l}- \\
6 \\
- \\
- \\
- \\
6\end{array}$ & $\begin{array}{r}- \\
17,1 \\
- \\
- \\
- \\
7,4\end{array}$ & $\begin{array}{l}1 \\
- \\
- \\
- \\
- \\
1\end{array}$ & $\begin{array}{r}2,4 \\
- \\
- \\
- \\
- \\
1,0\end{array}$ \\
\hline Mittlere Reife & $\begin{array}{l}\text { CDU / CSU } \\
\text { SPD } \\
\text { FDP } \\
\text { Grüne } \\
\text { Linke } \\
\text { insgesamt }\end{array}$ & $\begin{array}{r}4 \\
5 \\
1 \\
- \\
- \\
10\end{array}$ & $\begin{array}{r}9,5 \\
14,3 \\
25,0 \\
- \\
- \\
12,3\end{array}$ & $\begin{array}{l}1 \\
5 \\
1 \\
1 \\
1 \\
9\end{array}$ & $\begin{array}{r}2,4 \\
21,7 \\
8,3 \\
7,1 \\
12,5 \\
9,1\end{array}$ \\
\hline Abitur & $\begin{array}{l}\text { CDU / CSU } \\
\text { SPD } \\
\text { FDP } \\
\text { Grüne } \\
\text { Linke } \\
\text { insgesamt }\end{array}$ & $\begin{array}{r}38 \\
22 \\
3 \\
- \\
- \\
63\end{array}$ & $\begin{array}{r}90,5 \\
62,9 \\
75,0 \\
- \\
- \\
77,8 \\
\end{array}$ & $\begin{array}{r}36 \\
16 \\
11 \\
12 \\
7 \\
82\end{array}$ & $\begin{array}{l}85,7 \\
69,6 \\
91,7 \\
85,7 \\
87,5 \\
82,8 \\
\end{array}$ \\
\hline ohne Angabe, unklar & $\begin{array}{l}\text { CDU / CSU } \\
\text { SPD } \\
\text { FDP } \\
\text { Grüne } \\
\text { Linke } \\
\text { insgesamt }\end{array}$ & $\begin{array}{l}- \\
2 \\
- \\
- \\
- \\
2\end{array}$ & $\begin{array}{r}- \\
5,7 \\
- \\
- \\
- \\
2,5\end{array}$ & $\begin{array}{l}4 \\
2 \\
- \\
1 \\
- \\
7\end{array}$ & $\begin{array}{r}9,5 \\
8,7 \\
- \\
7,1 \\
- \\
7,1\end{array}$ \\
\hline $\begin{array}{l}\text { Studium } \\
\text { (Lehrerseminar, Fachhoch- } \\
\text { schule, Hochschule, Univer- } \\
\text { sität, Akademie der Arbeit) }\end{array}$ & $\begin{array}{l}\text { CDU / CSU } \\
\text { SPD } \\
\text { FDP } \\
\text { Grüne } \\
\text { Linke } \\
\text { insgesamt }\end{array}$ & $\begin{array}{r}33 \\
24 \\
2 \\
- \\
- \\
59\end{array}$ & $\begin{array}{r}78,6 \\
68,6 \\
50,0 \\
- \\
- \\
72,8\end{array}$ & $\begin{array}{r}34 \\
16 \\
10 \\
11 \\
8 \\
79\end{array}$ & $\begin{array}{r}80,9 \\
69,6 \\
83,3 \\
78,6 \\
100,0 \\
79,8\end{array}$ \\
\hline $\begin{array}{l}\text { insgesamt } \\
\text { absolut }=100 \text { Prozent } \\
\text { absolut }=100 \text { Prozent } \\
\text { absolut }=100 \text { Prozent } \\
\text { absolut }=100 \text { Prozent } \\
\text { absolut }=100 \text { Prozent }\end{array}$ & $\begin{array}{l}\text { CDU / CSU } \\
\text { SPD } \\
\text { FDP } \\
\text { Grüne } \\
\text { Linke } \\
\text { alle Parteien }\end{array}$ & $\begin{array}{r}42 \\
35 \\
4 \\
- \\
- \\
81\end{array}$ & & $\begin{array}{r}42 \\
23 \\
12 \\
14 \\
8 \\
99\end{array}$ & \\
\hline $\begin{array}{l}\text { Rundungsdifferenzen sind m } \\
\text { a Jeweils der höchste erlangt } \\
\text { b Zu Beginn der Wahlperioc } \\
\text { Quelle: Eigene Zusammenst }\end{array}$ & $\begin{array}{l}\text { oglich. } \\
\text { Bildungsabs } \\
\text { e (konstituier } \\
\text { llung. }\end{array}$ & itzun & & & \\
\hline
\end{tabular}




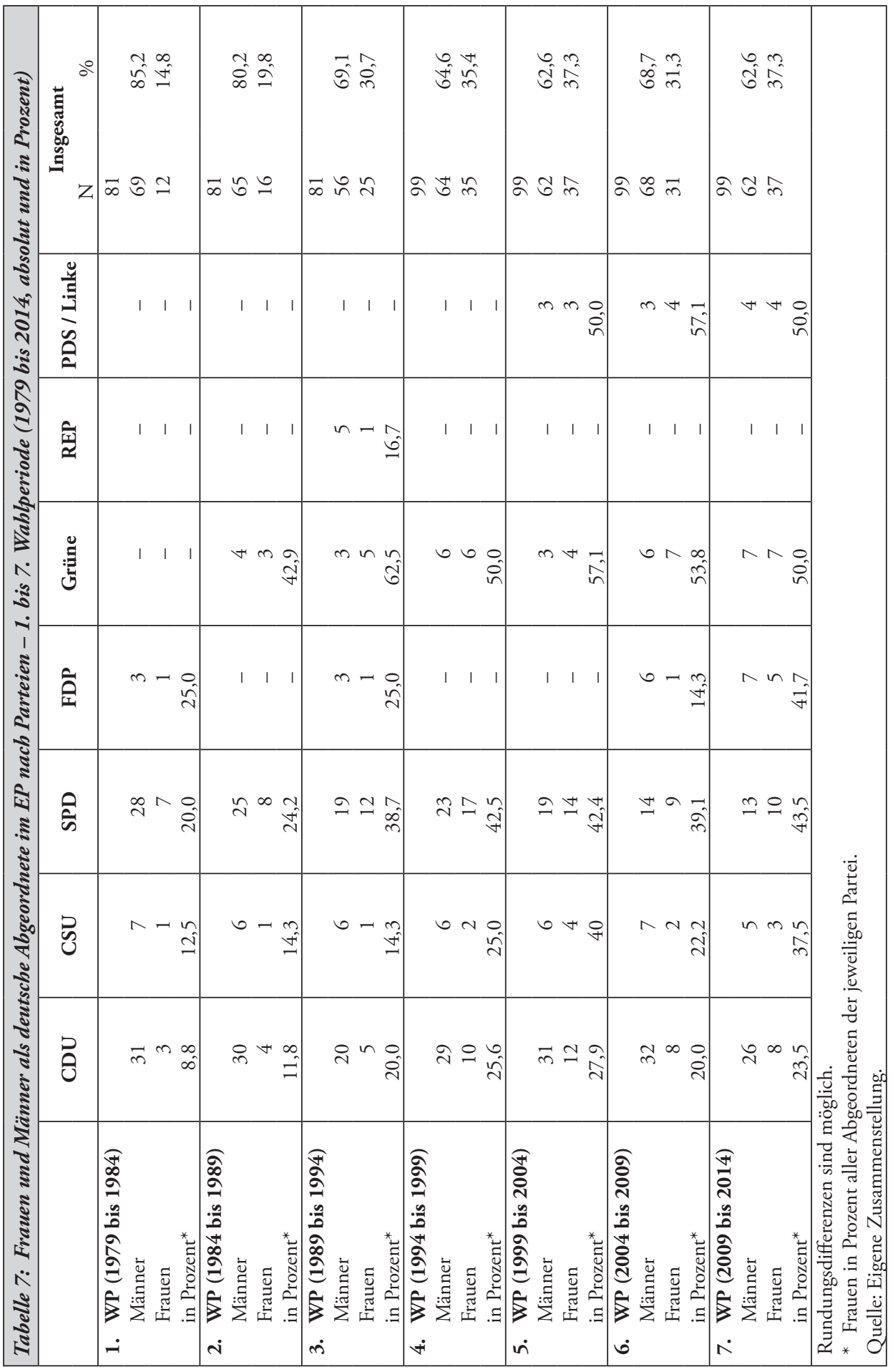




\section{Parlamentarische Erfahrungen der EP-Abgeordneten}

Nur wenige Politiker, die in das EP gewählt wurden, verfügten nicht über langjährige Erfahrung im politischen Raum. ${ }^{27}$ Dem Mandat gingen in beachtlichem Umfang parlamentarische Erfahrungen in Landesparlamenten und / oder im Bundestag voraus (vgl. Tabelle 8). Nachdem die Nachwirkung der Delegation von Abgeordneten des Bundestages in das bis 1979 nicht direkt gewählte EP sich minimiert hatte, verfügten seit der 3. Wahlperiode konstant etwa 30 Prozent der EP-Abgeordneten über eine parlamentarische Sozialisierung in Landesparlamenten oder im Bundestag (in der ersten und zweiten Wahlperiode lag der Anteil noch bei 64,2 Prozent beziehungsweise 44,4 Prozent). Fasst man die parlamentarischpolitische Sozialisierung in Landesparlamenten und Bundestag mit der im EP in vorangegangenen Wahlperioden erlangten Parlamentserfahrung zusammen, besaßen die MdEP bis zur gegenwärtigen 7. Wahlperiode in beachtlichem Umfang parlamentarische (Vor-)Erfahrungen: stets über 60 Prozent, in vier Wahlperioden sogar über 75 Prozent der Abgeordneten.

Bereits diese Daten lassen darauf schließen, dass das EP alles andere als ein Ort ist, um gealterten Parlamentariern und verdienten Politikern einen würdigen Abschluss für ihre politische Karriere zu bieten, eine letzte Wirkungsstätte mit moderaten Anforderungen und geringem Einfluss. ${ }^{28}$ Beide Datenreihen - die der parlamentarischen Vorerfahrung in Landesparlamenten und im Bundestag sowie die Kontinuität der Mandate im EP - zeigen, dass das EP-Mandat für viele Parlamentarier, die als Berufspolitiker agierten, keine kurzfristige Zwischen- oder Endstation war, sondern eine wichtige Etappe (mitunter ein berufspolitischer Höhepunkt) einer längeren parlamentarischen Karriere darstellte. Das EP wurde dabei - nach Möglichkeit - als institutionelle Basis für ein längeres politisches Engagement genutzt. Es diente für die deutschen MdEP jedoch nicht in nennenswertem Umfang als politisch-parlamentarische Zwischenstation für höhere politische Ambitionen und Funktionen (unter anderem Regierungsfunktionen, Funktionen in der EU). ${ }^{29}$

27 Zu den vielfältigen vorparlamentarischen politischen Erfahrungen zählen in der Regel verschiedene Parteifunktionen und kommunalpolitische Mandate, gelegentlich finden sich Tätigkeiten in europäischen Einrichtungen, in europa-lobbyistischen Agenturen oder in politischen oder politiknahen Institutionen mit europa-politischem Schwerpunkt. Auf diese Karrierestationen wie auch auf Funktionen in der jeweiligen Partei kann hier jedoch ebenso wenig eingegangen werden wie auf kommunalpolitische Erfahrungen und Stationen, die viele EP-Mitglieder vorweisen können. Vgl. Karlheinz Reif / Hermann Schmitt / Klaus Schubert, a.a.O. (Fn. 5), S. 341 f.; Rudolf Hrbek I Carl-Christoph Schweitzer, a.a.O. (Fn. 5), S. 4 f.

28 Gleichwohl lässt sich ein solcher Eindruck nicht immer von der Hand weisen, wenn zum Beispiel das EP-Mandat des langjährigen Bayerischen Ministerpräsidenten Alfons Goppel (geboren 1905) in der ersten Wahlperiode (1979 bis 1984) betrachtet wird oder in der 7. Wahlperiode die Mandatierung von Lothar Bisky. Eine Variante zu dieser politischen „Altersversorgung“ bildet immer noch die „Abfindung“ mit einem EP-Mandat, wenn innerparteiliche Funktionen umbesetzt oder Parlamentsmandate anderweitig vergeben werden (die EP-Mandate von Reinhard Bütikofer und Werner Schulz weisen in diese Richtung). Als Variante solcher „Versorgungsmandate“ mag die Mandatierung in Form einer parlamentarisch-politische "Zwischenlagerung“ angesehen werden, bevor eine Rückkehr in das politische System der Bundespolitik möglich wird (zum Beispiel Cem Özdemir oder Sarah Wagenknecht). Ausführlich zum „Verbleib“von in Deutschland gewählten Europa-Abgeordneten vgl. den weiteren Beitrag von Peter Rütters in diesem Heft der ZParl.

29 Für andere Verläufe politischer Karrieren vgl. Richard Corbett / Francis Jacobs / Michael Shackleton, a.a.O. (Fn. 1), S. 59 ff.; ferner Benjamin Höhne, a.a.O. (Fn. 19), S. 165 f. 


\begin{tabular}{|c|c|c|c|c|c|c|c|c|c|c|c|c|}
\hline $\begin{array}{l}3 \\
\frac{3}{3} \\
3 \\
\frac{3}{3}\end{array}$ & 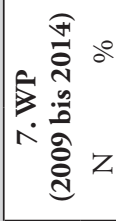 & $\begin{array}{l}\Rightarrow \stackrel{d}{*} \\
\Rightarrow \stackrel{\sim}{\sim}\end{array}$ & है & $\stackrel{m}{m}$ & $\vec{n}$ & $\vec{\infty}$ & $\begin{array}{l}0 \\
\infty \\
\text { in }\end{array}$ & $\stackrel{\infty}{\underbrace{0}}$ & $\curvearrowright$ & 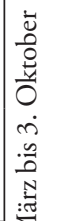 & 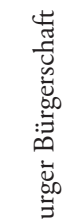 & 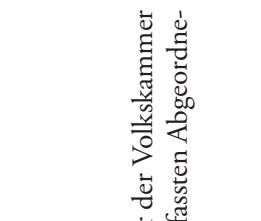 \\
\hline 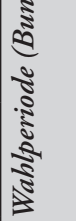 & 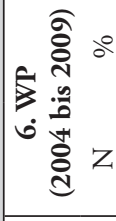 & $\begin{array}{l}\stackrel{\sim}{n} \stackrel{\infty}{\sim} \\
\cong \infty\end{array}$ & $\cong$ & तै & $\stackrel{\circ}{\forall}$ & $\vec{\infty}$ & $\begin{array}{l}\hat{\sigma} \\
\hat{\sigma}\end{array}$ & $\stackrel{\infty}{\infty}$ & え & 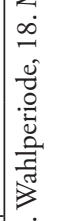 & 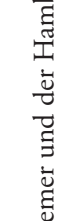 & 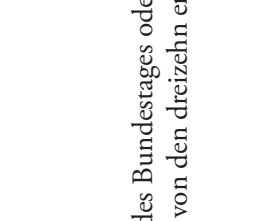 \\
\hline 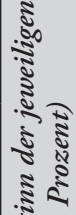 & 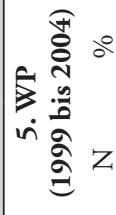 & 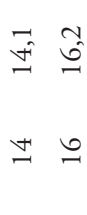 & $\vec{f}$ & $\begin{array}{l}n \\
\infty \\
\infty^{n}\end{array}$ & in & $\vec{\sigma}$ & $\hat{R}$ & $\stackrel{\infty}{\Omega}$ & え & 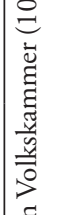 & 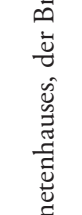 & 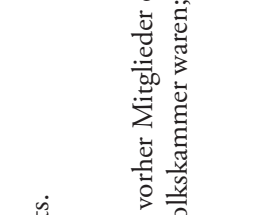 \\
\hline 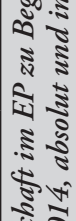 & 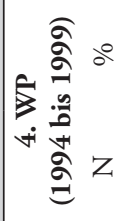 & 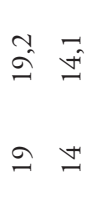 & $\exists$ & $\begin{array}{l}n \\
\hat{n}^{2}\end{array}$ & $\vec{n}$ & $\vec{n}$ & $\begin{array}{l}n \\
\substack{++\infty}\end{array}$ & $\hat{\theta}$ & ๙ & 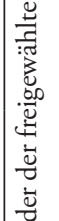 & 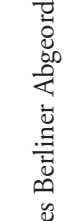 & 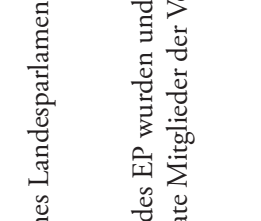 \\
\hline 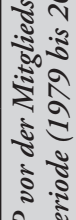 & 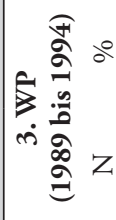 & $\begin{array}{l}\stackrel{\infty}{+} \stackrel{0}{ \pm} \\
\simeq \stackrel{n}{\simeq}\end{array}$ & 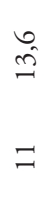 & $\begin{array}{l}W^{+} \\
\infty\end{array}$ & $\stackrel{\vartheta}{\forall}$ & ช̂ & $\begin{array}{l}0 \\
n \\
n\end{array}$ & $\hat{\sigma}$ & $\infty$ & 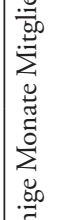 & 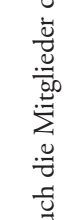 & 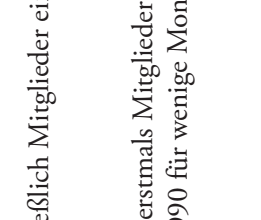 \\
\hline 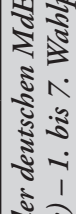 & 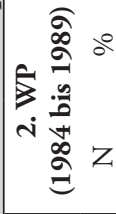 & 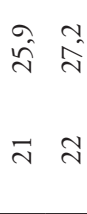 & $\begin{array}{l}\stackrel{n}{\infty} \\
\stackrel{\infty}{\infty}\end{array}$ & $\underset{f}{*}$ & $\hat{n}$ & ֶु & $\vec{\sigma}$ & $\begin{array}{l}\stackrel{0}{2} \\
\text { Ð }\end{array}$ & $\infty$ & 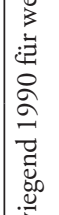 & 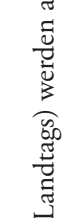 & 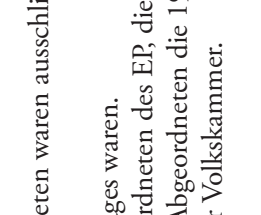 \\
\hline 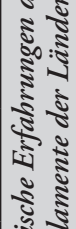 & 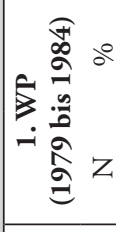 & $\begin{array}{l}\tilde{y} \\
\stackrel{f}{m} \\
\tilde{m} \\
\tilde{m}\end{array}$ & $\stackrel{\circ}{\vec{v}}$ & $\stackrel{\sim}{f^{f}}$ & $\tilde{\tilde{v}}$ & $\stackrel{\circ}{\vec{i}}$ & $\stackrel{\infty}{\stackrel{0}{\Omega}}$ & 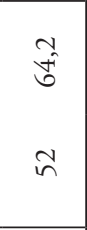 & $\infty$ & 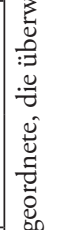 & 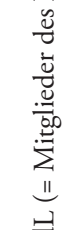 & 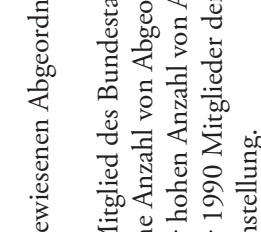 \\
\hline 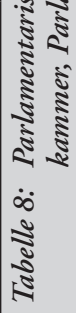 & & 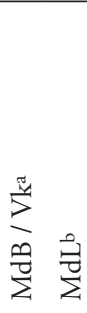 & 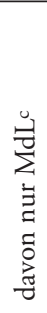 & 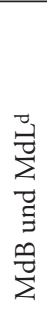 & 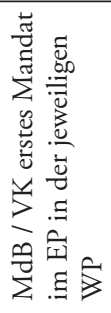 & 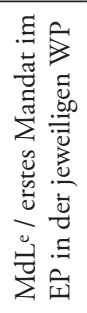 & 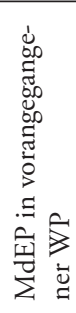 & 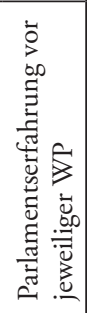 & 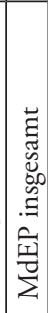 & 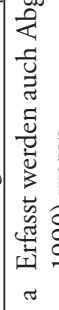 & 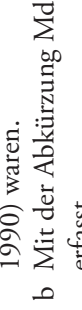 & 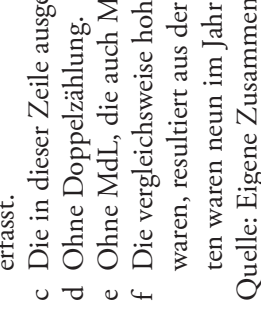 \\
\hline
\end{tabular}


Beim EP-Mandat handelt es sich jedoch (in der Regel) nicht um einen parlamentarischen „Joker“, der nach Belieben für ein Landtags- oder Bundestagsmandat „eingetauscht“ werden könnte. Der Wechsel vom EP in ein Landesparlament erfolgte nur äußerst selten; hingegen kam die Annahme eines Bundestagsmandats nach einer Zeit im EP ab und an vor, insgesamt war aber auch dies eher eine Ausnahme. Ein Wechsel zwischen Landes-, Bundes- und Europäischem Parlament erfolgte überwiegend als Aufstieg in das EP, das eine andere Art von Spezialisierung und parlamentarischer Kontroll- und Gestaltungsfunktionen verlangt und vermittelt als die Landesparlamente und der Bundestag.

Eine Durchlässigkeit der politischen Ebenen besteht für EP-Abgeordnete faktisch nur dann, wenn die im Europäischen Parlament erworbenen Kompetenzen für Regierungsaufgaben (vor allem in Landesregierungen) dienlich sein können. Eine etablierte Zirkulation von Abgeordneten zwischen den Parlamenten (und in die Regierungen) des europäischen Mehrebenensystems lässt sich dennoch nicht nachweisen.

\section{Regierungserfahrung von Abgeordneten des EP}

Eine Anzahl ehemaliger Mitglieder von Bundes- und Landesregierungen wurden mit mehr oder weniger geringem Abstand zum Ende ihres Regierungsamtes Mitglied des EP (vgl. Tabelle 9). Die beachtlichen Anteile ehemaliger Regierungsmitglieder insbesondere in der ersten und zweiten Wahlperiode des EP (16 Prozent beziehungsweise 9,8 Prozent der EP-Mitglieder) täuschen etwas hinsichtlich der Attraktivität eines Mandats für diese Gruppe von Berufspolitikern. Insbesondere in der ersten Wahlperiode finden sich mit Willy Brandt ein Alt-Bundeskanzler und mit Heinz Kühn und Alfons Goppel zwei nach sehr langer Amtszeit erst 1978 zurückgetretene Ministerpräsidenten sowie mit Kai-Uwe von Hassel, Katharina Focke und Hans Katzer drei ehemalige Bundesminister (vor dem Ministeramt war von Hassel bereits von 1954 bis 1963 Ministerpräsident von Schleswig-Holstein gewesen). Hinzu kommen noch sieben MdEP mit früherem Mandat als Landesminister oder Amt als Staatssekretär in einem Bundesland, von denen zumindest Rudi Arndt und Wilhelm F.T. Hahn über eine überregionale politischen Bekanntheit verfügten. Die europa-parlamentarische Präsenz dieser politischen Prominenten sollte - wie erwähnt - vor allem dazu dienen, für die erste Direktwahl des EP eine breite öffentliche Beachtung und Akzeptanz zu erreichen. Folglich schieden die meisten von ihnen noch während oder mit dem Ende der Wahlperiode aus dem EP aus.

Von den Bundespolitikern fand sich nur die relativ junge Katharina Focke (bei der Wahl 197956 Jahre) auch in der nächsten Wahlperiode im EP. Eine größere Kontinuität zeigte sich bei den MdEP, die vorher Landesminister oder Staatssekretäre waren: Sechs von sieben dieser Abgeordnetengruppe aus der ersten Wahlperiode gehörten dem EP auch in der zweiten Wahlperiode an und zwei noch in der dritten. Das EP bietet offensichtlich eine Perspektive für Politiker, die ihr Amt in einer Landesregierung aufgeben mussten. Das zeigte sich auch in den folgenden Wahlperioden: Mit Ausnahme eines Landespolitikers (Rudolf Schieler), der in der ersten Wahlperiode MdEP war, hatten alle ehemaligen Minister und Staatssekretäre von Landesregierungen mindestens zwei (acht), manche drei (sechs) und einer sogar (bisher) vier Wahlperioden lang ein EP-Mandat. 30

30 Insgesamt hatten in den sieben Wahlperioden 19 ehemalige Minister oder Staatssekretäre von Landesregierungen ein Mandat im EP. Drei von diesen 19 erlangten erst in der 7. Wahlperiode ein 


\begin{tabular}{|c|c|c|c|c|c|c|c|}
\hline $\begin{array}{r}\text { belle 9: Regiern } \\
\text { absolut }\end{array}$ & $\begin{array}{l}\text { fahrung } \\
\text { in Proze }\end{array}$ & deutsc & $d E P$ & 7. $\mathrm{Wa}$ & de & & \\
\hline $\begin{array}{l}\text { Regierungs- } \\
\text { funktion }^{\mathrm{a}}\end{array}$ & $\begin{array}{c}\text { 1. WP } \\
\text { (1979 bis } \\
1984)\end{array}$ & $\begin{array}{c}\text { 2. WP } \\
\text { (1984 bis } \\
1989)\end{array}$ & $\begin{array}{c}\text { 3. WP } \\
\text { (1989 bis } \\
1994)\end{array}$ & $\begin{array}{c}\text { 4. WP } \\
\text { (1994 bis } \\
1999)\end{array}$ & $\begin{array}{l}\text { 5. WP } \\
\text { (1999 bis } \\
2004)\end{array}$ & $\begin{array}{c}\text { 6. WP } \\
(2004 \text { bis } \\
2009)\end{array}$ & $\begin{array}{c}\text { 7. WP } \\
\text { (2009 bis } \\
2014)\end{array}$ \\
\hline & $\%$ & $\mathrm{~N}$ & $\mathrm{~N}$ & $\mathrm{~N}$ & $\mathrm{~N}$ & $\%$ & $\mathrm{~N}$ \\
\hline $\begin{array}{l}\text { Bundeskanzler / Mi- } \\
\text { nisterpräsident DDR }\end{array}$ & $1^{\mathrm{c}} \quad 1,2$ & - & - & - & $1^{\circ} \quad 1,0$ & - & - \\
\hline $\begin{array}{l}\text { Ministerpräsident } \\
\text { (Bundesland)b }\end{array}$ & $2^{\mathrm{d}} \quad 2,5$ & - & - & $1^{\mathrm{k}} \quad 1,0$ & $1 \mathrm{p} \quad 1,0$ & $1^{\mathrm{r}} \quad 1,0$ & - \\
\hline $\begin{array}{l}\text { Bundesminister / } \\
\text { Staatssekretär }\end{array}$ & & $1 \mathrm{~g} \quad 1,2$ & - & $1^{\mathrm{m}} \quad 1,0$ & - & - & - \\
\hline $\begin{array}{l}\text { Landesminister / } \\
\text { Staatssekretär }\end{array}$ & & $7^{\text {h }} \quad 8,6$ & $4 \mathrm{i} \quad 4,9$ & $4^{\mathrm{n}} \quad 4,0$ & $7 q \quad 7,1$ & $6^{s} \quad 6,1$ & $7^{\mathrm{t}} \quad 7,1$ \\
\hline insgesamt & $13 \quad 16,0$ & $8 \quad 9,8$ & $4 \quad 4,9$ & $6 \quad 6,1$ & 9,1 & 7,1 & 7,1 \\
\hline MdEP insgesamt & 81 & 81 & 81 & 99 & 99 & 99 & 99 \\
\hline $\begin{array}{l}\text { a Bei mehreren Funkti } \\
\text { b Ministerpräsident ein } \\
\text { (Berlin), Bürgermeist } \\
\text { c Von } 1979 \text { bis } 1983 \text { g } \\
\text { diesem Mandat war e } \\
\text { d Heinz Kühn (SPD) w } \\
1962 \text { bis } 1978 \text { Minis } \\
\text { e Katharina Focke (SPD } \\
\text { nisterin für Jugend, } \\
\text { und Sozialordnung; } \\
\text { 1966) für Vertriebene } \\
\text { Holstein. } \\
\text { f Landesminister warer } \\
\text { am Main); Wilhelm } \\
\text { Staatssekretär in Rhei } \\
\text { Beamteter Staatssekre } \\
\text { Schön (CDU, 1974 b } \\
\text { g Katharina Focke, sieh } \\
\text { h Rudi Arndt (SPD), } \\
\text { (CDU), Hans-Joachi } \\
\text { Landesminister in Ba } \\
\text { i Horst Langes (CDU) } \\
\text { (SPD, } 1974 \text { bis } 1987 \\
\text { k Alfred Gomolka (CDL } \\
\text { m Wolfgang Ullmann Fe } \\
\text { n Willi Görlach (SPD), } \\
\text { Konrad Schwaiger (C } \\
\text { Langen (CDU) 1990 } \\
\text { o Hans Modrow (PDS) } \\
\text { p Alfred Gomolka (CD } \\
\text { q Willi Görlach (SPD), } \\
\text { (CDU), siehe n); net } \\
\text { 1999 Staatssekretär ir } \\
\text { r Alfred Gomolka (CD } \\
\text { s Werner-Josef Langen ( } \\
\text { Hans-Peter Mayer (CI } \\
\text { t Werner-Josef Langen } \\
\text { Helga Trüpel (Die Gr } \\
\text { Landesministerin in } \\
\text { Winkler (CDU), 200 } \\
\text { Quelle: Eigene Zusamn }\end{array}$ & 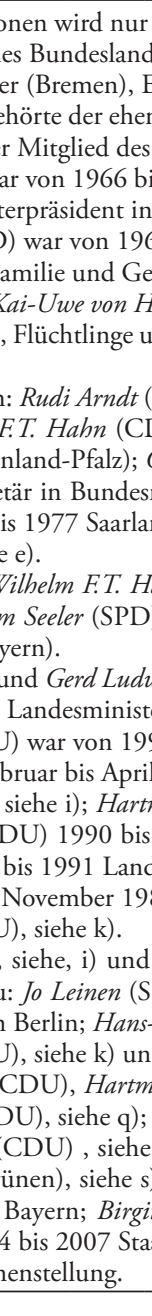 & 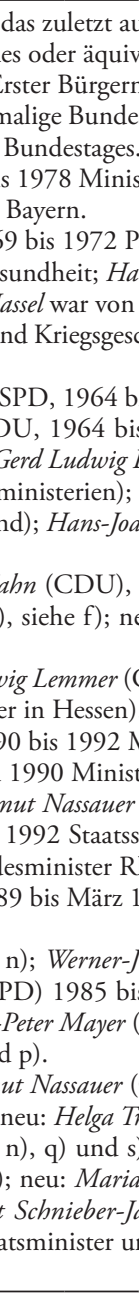 & $\begin{array}{l}\text { eübte Regi } \\
\text { ente Bezeic } \\
\text { ister (Ham } \\
\text { anzler (196 } \\
\text { rpräsident } \\
\text { lamentarisc } \\
\text { Katzer (CI } \\
963 \text { bis } 196 \\
\text { ädigte, von } \\
1972 \text { in He } \\
1978 \text { Bader } \\
\text { mmer (CD } \\
\text { udolf Schiel } \\
\text { im Seeler ( } \\
\text { orst Langes } \\
\text { : Fritz Pirk } \\
\text { JU) siehe f } \\
\text { nisterpräsic } \\
\text { ohne Gesc } \\
\text { CDU) Nov } \\
\text { cretär im X } \\
\text { inland-Pfal } \\
90 \text { Ministe } \\
\text { ef Langen ( } \\
1994 \text { Land } \\
\text { DU) 1991 } \\
\text { DU), siehe } \\
\text { pel (Die Gr } \\
\text { Jo Leinen ( } \\
\text { Hohlmeier ( } \\
\text { ram (CDU } \\
1 \text { Chef der } \\
\end{array}$ & $\begin{array}{l}\text { ngsamt in } \\
\text { ing in den } \\
\text { g). } \\
\text { is } 1974) \text { W } \\
\text { Jordrhein-- } \\
\text { Staatssekret } \\
\text { war von } 1 \\
\text { undesmini } \\
4 \text { bis } 1963 \\
\text { n; } 1972 \text { bis } \\
\text { ürttembers } \\
962 \text { bis } 19 \\
\text { SPD, } 1966 \\
\text {, } 1967 \text { bis } \\
\text { DU), Gerd } \\
\text { CSU, } 1964 \\
\text { ld h); Fritz } \\
\text { von Meck } \\
\text { tsbereich in } \\
\text { per } 1990 \text { bi } \\
\text { chaftsmini } \\
\text { sident der } \\
\text { U), Hartm } \\
\text { inister im } \\
1994 \text { Staat } \\
\text { und q); Jo } \\
\text { n), } 1991 \text { b } \\
\text { U) und Ha } 1993 \text { b } \\
\text { 2001 bis } 2 \\
\text { tskanzlei in } \\
\end{array}$ & $\begin{array}{l}\text { illy Brandt ( } \\
\text { Vestfalen; A } \\
\text { ärin und vo } \\
965 \text { bis } 196 \\
\text { ter zunächs } \\
\text { war er Mini } \\
1977 \text { Ober } \\
\text { ); Horst La } \\
66 \text { Nordrhe } \\
\text { bis } 1972 \text { B } \\
1978 \text { Senat } \\
\text { Ludwig Len } \\
\text { bis } 1966 \text { St } \\
\text { Pirkl (CSU) } \\
\text { enburg-Vor } \\
\text { der Regiert } \\
\text { April } 1991 \\
\text { tterium in S } \\
\text { DDR. } \\
\text { ut Nassauer } \\
\text { aarland; In } \\
\text { sekretär in } \\
\text { Leinen (SPD } \\
\text { s } 1995 \text { Sena } \\
\text { as-Peter Ma } \\
\text { s } 1998 \text { Staa } \\
\text { D08 Senato }\end{array}$ & $\begin{array}{l}\text { ) dem EP } \\
\text { soppel (C } \\
972 \text { bis } 19 \\
\text { undesmini } \\
\text { r Verteidig } \\
\text { präsident v } \\
\text { germeister } \\
\text { (CDU, } \\
\text { Xestfalen; } \\
\text { n-Württem } \\
\text { n Hambur } \\
\text { (CDU), } \\
\text { sekretär, } 1 \\
\text { he h); neu: } \\
\text { nmern. } \\
\text { von Hans } \\
\text { indesminis } \\
\text { sen-Anhal } \\
\text { ing } \\
\text { ingo Schmi } \\
\text { in in Bretärin, } 1 \\
\text { in Hambs }\end{array}$ & 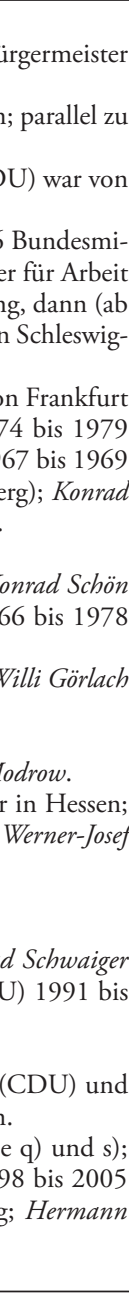 \\
\hline
\end{tabular}


Dass mit Hans Modrow (SED / PDS / Die Linke) ein ehemaliger (kurzzeitiger) Ministerpräsident der DDR für eine Wahlperiode Abgeordneter im EP war, kann ebenso als vereinigungsbedingte Besonderheit angesehen werden wie das EP-Mandat von Wolfgang Ullmann, der als Minister ohne Geschäftsbereich 1990 der Modrow-Regierung angehörte. Als alternativer politischer Karriereweg mag das Abgeordneten-Mandat von Alfred Gomolka (CDU) verstanden werden, der Anfang der 1990er Jahre für etwa 18 Monate Ministerpräsident in Mecklenburg-Vorpommern und ab 1994 für drei Wahlperioden Abgeordneter im EP war. Aber auch dieser Weg ins EP bestätigt, dass Regierungserfahrung vor allem von Mitgliedern von Landesregierungen - überwiegend von Ministern und Staatssekretären - in das EP eingebracht wird. Insgesamt ist dieser Kompetenztransfer jedoch gering, auch im Vergleich zu den Abgeordneten anderer EU-Mitgliedstaaten. ${ }^{31}$

\section{Dauer der Parlamentsmitgliedschaft}

Welche Relevanz ein Mandat im EP für die politische Karriere hat - ob es als eine kurze Durchgangsphase zu anderen Ämtern und Mandaten genutzt wurde, den Abschluss einer politisch-parlamentarischen Karriere darstellte, die überwiegend in anderen politischen Gremien (Landesparlament und/oder Bundestag, als Regierungsmitglied, in kommunalen Selbstverwaltungsgremien) stattfand, oder ob das EP zum zentralen Ort des eigenen politischen Wirkens wurde - lässt sich mit der folgenden Darstellung der Dauer der Parlamentsmitgliedschaft zwar nicht abschließend klären. Doch lassen sich mit einem Blick auf die Dauer der Parlamentsmitgliedschaft zumindest einige Hinweise geben, ob ein Mandat im EP eher beiläufig wahrgenommen wurde oder als Ausgangspunkt für europapolitische Mitwirkung und Mitentscheidung diente. 32

Das EP zeichnet sich - wie alle repräsentativ-demokratischen Parlamente - durch zwei parallele Entwicklungen aus: eine (mäßige) kontinuierliche „Erneuerung“ der Abgeordneten und eine relativ lange Verweildauer eines beachtlichen Teils der Parlamentarier.

Abgesehen vom parteiinternen Austausch von Mandatsträgern (siehe unten) wurde die „Erneuerung“ der Abgeordneten durch drei Faktoren beeinflusst: (1) die Veränderung der insgesamt verfügbaren Mandate; (2) Veränderungen der im EP vertretenen Parteien; (3) Veränderungen der von den einzelnen Parteien in den Wahlen erzielten Mandate.

(1) In der ersten Wahlperiode erfolgte der Zugang von neuen Abgeordneten (80,2 Prozent) vor allem, weil die Anzahl der EP-Mandate von 36 auf 81 vergrößert wurde (plus 125 Prozent). Etwas schwächer findet sich dieser Effekt in der 4. Wahlperiode, als durch die Berücksichtigung der neuen Bundesländer die Anzahl der Sitze um 18 auf 99 erhöht wurde

EP-Mandat, eine Abgeordnete war erst seit der 6. Wahlperiode MdEP und drei waren es seit der 5. Wahlperiode. Für insgesamt sieben MdEP der laufenden Wahlperiode kann sich die MdEP-Zeit um mindestens eine Wahlperiode verlängern.

31 Vgl. Richard Corbett / Francis Jacobs / Michael Shackleton, a.a.O. (Fn. 1), S. 54 - 58; ferner Emil J. Kirchner, a.a.O. (Fn. 1), S. 45 ff., S. 50 ff.

32 Eine tabellarische Darstellung zur Dauer der Mitgliedschaft im Europäischen Parlament (1979 bis 2014) findet sich in Peter Rütters, „,Verbleib“ von in Deutschland gewählten Europa-Abgeordneten “ in diesem Heft der ZParl. Die Tabelle 1 dieses Beitrags (S. 787) liegt der folgenden Darstellung zugrunde. 
(22,2 Prozent). Rechnet man diesen Effekt heraus, lag die Wiederwahlquote von der 2. bis zur 7. Wahlperiode etwa bei 60 bis 70 Prozent.

(2) Mit etwas geringerer Wirkung hatten Veränderungen der im EP vertretenen Parteien unmittelbaren Einfluss auf den Zugang und das Ausscheiden von Parlamentariern. In der 2. Wahlperiode (1984 bis 1989) umfasste der Eintritt der Grünen in das EP mit sieben MdEP bereits 8,6 Prozentpunkte (27,8 Prozent) der insgesamt 30,9 Prozent (absolut: 25) neuen EP-Mitglieder. In der folgenden 3. Wahlperiode (1989 bis 1994) machten die Abgeordneten der neu (REP) und der wieder (FDP) im EP vertretenen Parteien zusammen bereits 12,3 Prozentpunkte (25,6 Prozent; absolut zehn) von insgesamt 48,1 Prozent (39) der neuen EP-Mitglieder aus. Und in der Umkehrung bewirkte allein das Ausscheiden von FDP und REP mit dem Ende der 3. Wahlperiode (1994) eine Öffnung des Parlaments für neue Abgeordnete der etablierten Parteien in der 4. Wahlperiode. Von den 53 (53,5 Prozent) im Jahr 1994 neu in das EP gewählten Abgeordneten erhielten 18 durch die Erhöhung der Anzahl der Sitze sowie zehn durch das Ausscheiden von zwei Parteien einen Sitz im EP (28,3 Prozentpunkte beziehungsweise 52,8 Prozent). Der „reine“ Wechsel von Abgeordneten als Folge des Ausscheidens bisheriger Parlamentsmitglieder der weiterhin im EP vertretenen Parteien umfasste somit nur 25 Abgeordnete (25,2 Prozentpunkte beziehungsweise 47,2 Prozent).

(3) Die Veränderung des Anteils der Sitze, die einzelne Parteien im EP erlangen konnten, trug unmittelbar dazu bei, den Zugang für neue Parlamentarier zu beeinflussen. Beispielsweise büßte die CDU/CSU neun Mandate bei der EP-Wahl 1989 ein, die anderen Parteien zugute kamen; das waren 11,1 Prozentpunkte von 48,1 Prozent (absolut 39) aller neuen $\operatorname{MdEP}(23,1$ Prozent).

Neben diesen durch Strukturveränderungen hervorgerufenen Modifikationen in der Zusammensetzung der EP-Abgeordneten und der Verweildauer im Parlament folgten der Zugang neuer und das Ausscheiden alter MdEP vor allem den Vorentscheidungen der Parteien bei der Aufstellung der Kandidatenlisten (der Nominierungen auf sicheren Listenplätzen).33 Exemplarisch lassen sich solche Entscheidungen bei der SPD zeigen, als sie bei der EP-Wahl 2004 nur noch 23 Sitze erreichte, zehn weniger als bei der vorangegangenen Wahl im Jahr 1999. Die Listenabsicherung bisheriger SPD-Kandidaten minimierte den Spielraum für eine Personalerneuerung auf einen Abgeordneten (Vural Öger). ${ }^{34}$ Erst bei der folgenden Wahl 2009 wurde eine personelle Erneuerung durch die Nominierung neuer Kandidaten auf aussichtsreichen Plätzen der SPD-Wahlliste vorbereitet, so dass trotz der unveränderten Anzahl der Parlamentssitze zehn von insgesamt 23 Abgeordneten neu in das EP einziehen konnten (vgl. Tabelle 10) und die Phase personal-innovativer Stagnation der 5. und 6. Wahlperiode beendet wurde. 35

Kontrastierend zu dem mitunter umfangreichen Personalwechsel von Wahlperiode zu Wahlperiode dominiert die Kontinuität unter den EP-Abgeordneten. Dass noch im Parlament der 7. Wahlperiode zwei Abgeordnete sitzen (Elmar Brok, Hans-Gert Pöttering - beide

33 Zur Nominierungspraxis der Parteien für die Europawahlen vgl. Benjamin Höhne, a.a.O. (Fn. 19).

34 Zur Nominierung von Vural Öger vgl. Oskar Niedermayer, Europa als Randthema: Der Wahlkampf und die Wahlkampfstrategien der Parteien, in: ders. / Hermann Schmitt (Hrsg.), Europawahl 2004, Wiesbaden 2005, S. 39 - 75, S. 44; ferner Benjamin Höhne, a.a.O. (Fn. 19), S. 164 f.

35 Zur Kandidatenaufstellung für die Europawahl 2009 vgl. Benjamin Höhne, a.a.O. (Fn. 19), S. 191 f., S. 273 ff., S. 303 f. Diese personelle Erneuerung musste sich innerparteilich gegen manche Widerstände von etablierten EP-Abgeordneten durchsetzen, die durchaus bereit und willens waren, ihr EP-Mandat aufrechtzuerhalten. 


\begin{tabular}{|c|c|c|c|c|}
\hline$\Xi$ & 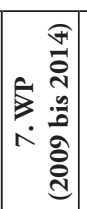 & 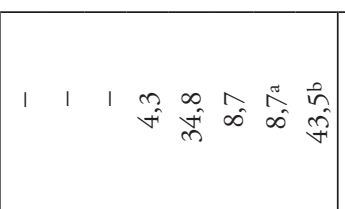 & $\stackrel{8}{2}$ & \\
\hline 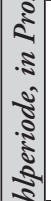 & 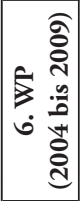 & 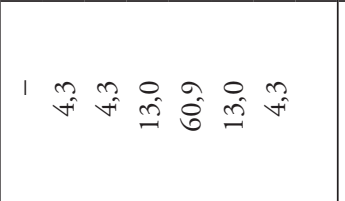 & $\stackrel{8}{8})$ & \\
\hline 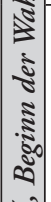 & 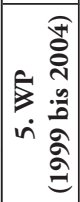 & $\vec{a} \vec{a} \tilde{\vec{N}} \approx \vec{f} \vec{I}$ & 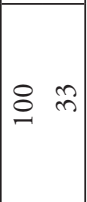 & \\
\hline $\begin{array}{c}0 \\
2 \\
-3 \\
\vdots \\
\hat{2} \\
2\end{array}$ & 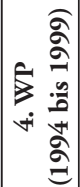 & 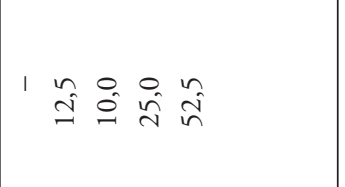 & $\stackrel{8}{8}$ & \\
\hline م. & 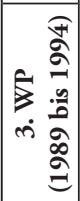 & 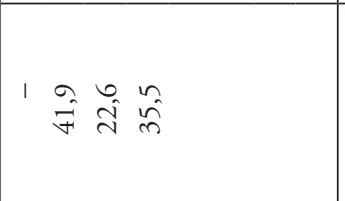 & $\stackrel{一}{\circ}$ & 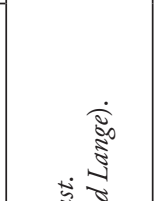 \\
\hline 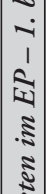 & 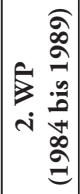 & $\overrightarrow{0} \hat{b} \tilde{d}$ & $\stackrel{8}{\circ}$ & 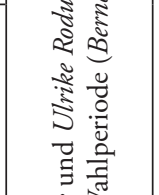 \\
\hline 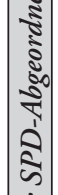 & 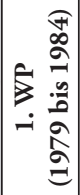 & $\mid \begin{array}{ll}0 \\
0 \\
\infty & \vec{a} \\
\infty & a\end{array}$ & 8 c & 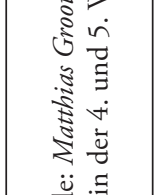 \\
\hline 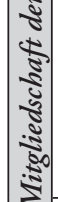 & 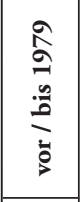 & 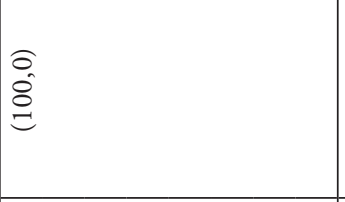 & & 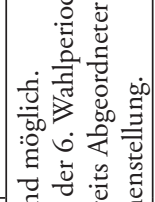 \\
\hline 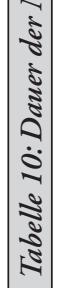 & & 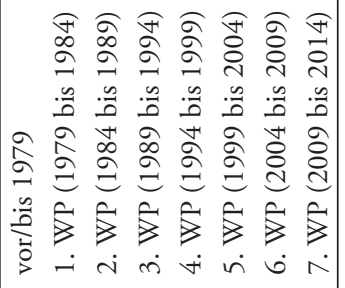 & 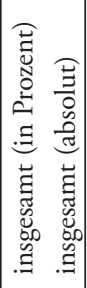 & 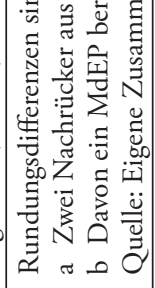 \\
\hline
\end{tabular}


CDU), die seit der 1. Wahlperiode kontinuierlich Parlamentsmitglieder sind, mag nur auf den ersten Blick als marginale Besonderheit gewertet werden. Wie in allen Parlamenten nimmt der politisch-administrative Einfluss von Abgeordneten in der Regel mit der Verweildauer zu. Eine entsprechende personelle Kontinuität baute sich seit der 1. Wahlperiode von Wahl zu Wahl auf und führte dazu, dass in den jüngeren Wahlperioden Abgeordnete vertreten waren/sind, die bis zu vier oder fünf Wahlperioden im EP saßen/sitzen.

Allein in den letzten beiden Wahlperioden gehörten 47,5 Prozent (47) beziehungsweise 35,4 Prozent (35) der Abgeordneten bereits zwei und mehr Wahlperioden dem EP an.

Deutlicher noch als die Gesamt-Tabelle zur Dauer der Mitgliedschaft ${ }^{36}$ zeigen die beiden Parteien-Tabellen für die SPD und die CDU/CSU (vgl. Tabelle 10 und Tabelle 11)37, dass die Parteien (und Parlamentarier) bestrebt sind, durch die personelle Kontinuität im EP die von den Abgeordneten gewonnenen europa-politischen und europa-parlamentarischen (institutionellen) Kompetenzen zu erhalten. Mandatsgewinne ergänzen diese Personalpolitik der Parteien, da sie die Chance für eine personelle „Erneuerung“ der Abgeordneten bieten, ohne in gleichem Umfang bisherigen Mitgliedern des EP das Mandat zu entziehen. Phasen der Stagnation oder der Verminderung der Anzahl der Mandate führen - wie erwähnt zunächst dazu, die personelle Innovation zu hemmen. Die CDU/CSU zeigt im Grunde ein ähnliches Muster personeller Erneuerung wie die Sozialdemokraten.

\section{Das Mandat im EP: hohe Kontinuität und europäische Politik als Beruf}

Generell weisen die hier tabellarisch zusammengefassten Daten auf Folgendes hin: (1) eine bemerkenswerte personelle Kontinuität bei den Abgeordneten, die einen ähnlichen Umfang hat wie im Bundestag und in den Landesparlamenten; (2) die berufspolitische Wahrnehmung des EP-Mandates; (3) eine politische Handlungsorientierung, die das EP als Institution politisch-parlamentarischer Mitwirkung und Mitgestaltung auffasst, so dass (4) das Mandat für das EP nicht (vorrangig) für einen kurzen Zwischenstopp einer eher bundespolitisch ausgerichteten Karriere dient, das EP nicht überwiegend als alimentierende Versorgungsstätte angesehen oder als Ort für ein gemächliches „Ausschleichen“ aus einem langen berufspolitischen Leben genutzt wird.

36 Vgl. Tabelle 1 in Peter Rütters, „,Verbleib“ von in Deutschland gewählten Europa-Abgeordneten“, S. 787 in diesem Heft der ZParl.

37 Angesichts der geringen Anzahl der Mandate und der Diskontinuität der Vertretung im EP lassen sich für die kleinen Parteien (FDP, Die Grünen und PDS/Die Linke) keine aussagekräftigen Tabellen erstellen. 


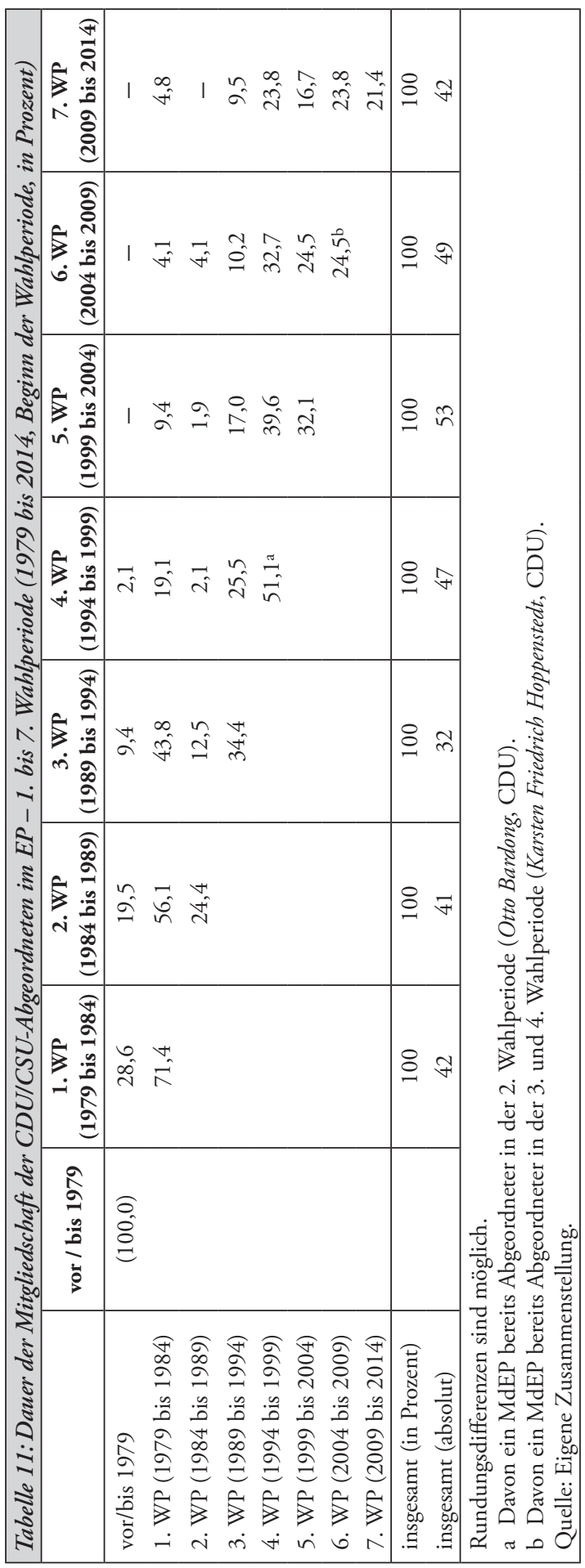

